\title{
High NA Microstepper Final Optical Design Report
}

\author{
R. Hudyma
}

September 24, 1999

U.S. Department of Energy

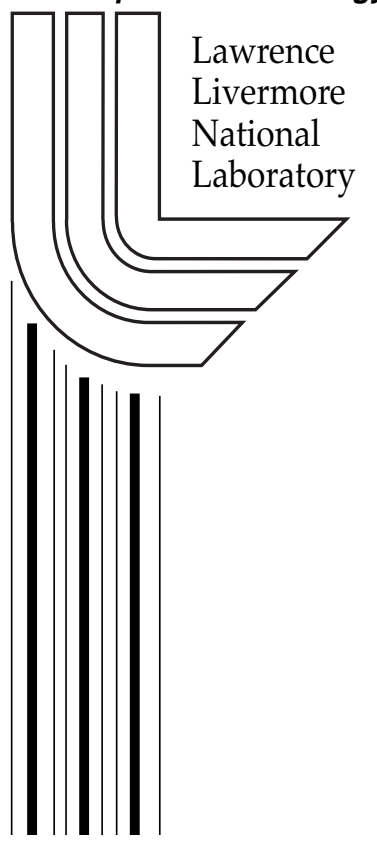




\section{DISCLAIMER}

This document was prepared as an account of work sponsored by an agency of the United States Government. Neither the United States Government nor the University of California nor any of their employees, makes any warranty, express or implied, or assumes any legal liability or responsibility for the accuracy, completeness, or usefulness of any information, apparatus, product, or process disclosed, or represents that its use would not infringe privately owned rights. Reference herein to any specific commercial product, process, or service by trade name, trademark, manufacturer, or otherwise, does not necessarily constitute or imply its endorsement, recommendation, or favoring by the United States Government or the University of California. The views and opinions of authors expressed herein do not necessarily state or reflect those of the United States Government or the University of California, and shall not be used for advertising or product endorsement purposes.

This work was performed under the auspices of the U. S. Department of Energy by the University of California, Lawrence Livermore National Laboratory under Contract No. W-7405-Eng-48.

This report has been reproduced directly from the best available copy.

Available electronically at http://www.doc.gov/bridge

Available for a processing fee to U.S. Department of Energy

And its contractors in paper from

U.S. Department of Energy

Office of Scientific and Technical Information

P.O. Box 62

Oak Ridge, TN 37831-0062

Telephone: (865) 576-8401

Facsimile: (865) 576-5728

E-mail: reports@adonis.osti.gov

Available for the sale to the public from

U.S. Department of Commerce

National Technical Information Service

5285 Port Royal Road

Springfield, VA 22161

Telephone: (800) 553-6847

Facsimile: (703) 605-6900

E-mail: orders@ntis.fedworld.gov

Online ordering: http://www.ntis.gov/ordering.htm

OR

Lawrence Livermore National Laboratory

Technical Information Department's Digital Library

http:/ / www.llnl.gov/tid/Library.html 


\section{Information Science \& Technology Program}

September 24, 1999

To: Distribution

From: Russ Hudyma

Subject: High NA microstepper final optical design report

\section{References}

1. R. Hudyma, "Projection Concepts for Advanced Microstepper," AMP Technical Memorandum M9801124, August 31, 1998.

2. J.Bokor and D.Shafer, "Design study for a high NA (>0.25) microstepper objective," LBL Technical Memorandum, September 18, 1998.

3. R. Hudyma, "Review of 4-6x Advanced Microstepper Optical Designs," CDR package, June 23, 1999.

\subsection{Introduction}

The development of a new EUV high NA small-field exposure tool has been proposed for obtaining mask defect printability data in a timeframe several years before beta-tools are available. The imaging system for this new Micro-Exposure Tool (MET), would have a numerical aperture (NA) of about 0.3, similar to the NA for a beta-tool, but substantially larger than the 0.10 NA for the Engineering Test Stand (ETS) and 0.088 NA for the existing 10x Microstepper. This memorandum discusses the development and summarizes the performance of the camera for the MET and includes a listing of the design prescription, detailed analysis of the distortion, and analysis demonstrating the capability to resolution $30 \mathrm{~nm}$ features under the conditions of partially coherent illumination.

\subsection{Overview of optical system}

The idea of employing a 2-mirror aspheric imaging system has been proposed in earlier reports. ${ }^{1,2}$ To achieve the largest possible field of view, the MET projection optics utilize a primary and secondary mirror whose radii are nearly the same (within $10 \%$ of each other). This enables the field curvature to be corrected to a value that approaches more sophisticated multi-mirror EUVL projection systems. Compared to the 10x imaging system using the same field size at the wafer, this "equal radii" concept reduces the longitudinal field curvature from $1.8 \mu \mathrm{m}$ to $0.05 \mu \mathrm{m}$. This $36 \mathrm{x}$ reduction in field curvature enables the depth of focus to accommodate subtle tilts of the wafer up to $\sim 1^{\circ}$. The result is depicted graphically in Figure 1. Fundamentally, the flat focal surface allows a tilted wafer plane to fall within the allowable depth of focus. Since imaging is controlled by the Scheimpflug condition ${ }^{1}$, the mask can be tilted by up to $\sim 5^{\circ}$, enabling the used of a reflective mask.

\footnotetext{
${ }^{1}$ The Scheimpflug condition states that the imaging properties of a centered optical system with a tilted object are preserved on a tilted image plane (ignoring distortion). For a system used at finite conjugates, the image plane tilt is the object plane tilt scaled by the reduction ratio.
} 


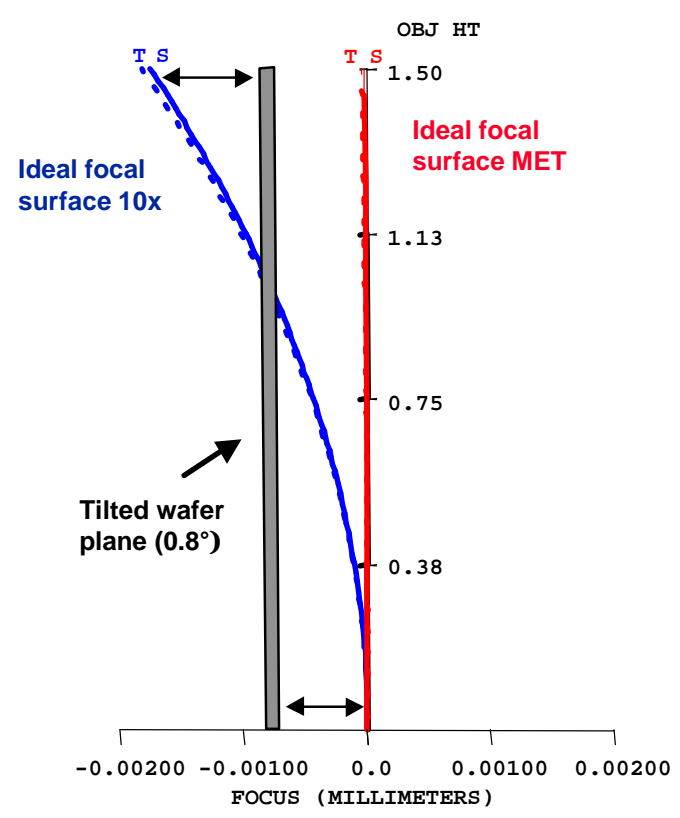

Figure 1. The principle feature of the MET design is the reduction in field curvature, which allows focus to be maintained across the entire tilted wafer plane. For reference, the ideal focal surface of the $10 x$ imaging system is shown in blue. The image formed at the wafer with the 10x camera would be outside depth of focus due to the curvature of field. The ideal focal surface of the MET camera is depicted in red. This focal surface is very flat, enabling a slightly tilted wafer to lie with the depth of focus, ensuring good imaging across the printed field.

Layouts of the final optical design with reflection and transmission masks are shown in Figure 2 and Figure 3, respectively. Table 1.0 summarizes the performance of the optical design relative to parameter goals. A large numerical aperture of 0.30 is attained by the use of a centered design, where the imaging bundles are centered on the optical axis. The centered design necessitates that the image passes through a hole in the primary mirror. Eccentric or off-axis pupil design forms are not feasible because the individual mirrors work at very fast conjugates. The residual aberrations simply grow too quickly to correct as the pupil moves off the optical axis. This fact forces the central obscuration on the exit pupil of the imaging system. To minimize obscured pupil area $(<10 \%)$, the image plane must be kept close to the primary mirror. This reduces the clearance between the back of the primary and wafer. The vertex thickness of the primary was set to $20 \mathrm{~mm}$ to provide ample substrate stiffness, leaving only $5 \mathrm{~mm}$ of clearance between the back of the primary and the wafer. This makes mechanical packaging of the primary difficult and precludes the use of a grazing-incidence focus system.

While the limited clearance makes the mechanical design more complicated, this issue is manageable. The working distance is, in fact, about the same as for contemporary DUV steppers. The final design shown in Figure 2 includes a proposed mirror substrate thickness to help visualize clearance at the wafer and how the illumination is brought onto the mask. The design is optimized to work at a $5 \mathrm{x}$ reduction across a rectangular field of view of $600 \mu \mathrm{m} \times 200 \mu \mathrm{m}$ at the wafer. While the field could be extended in the long dimension, the aspect ratio of 3:1 will help to simplify the design of the illumination system. The mask is tilted clockwise at $4.0^{\circ}$; the wafer has a corresponding counterclockwise tilt of $0.8^{\circ}$. This is the minimum tilt required to avoid interference between the incoming illumination and imaging bundle.

With a reflection mask, the composite RMS wavefront error across a $600 \mu \mathrm{m}$ x $200 \mu \mathrm{m}$ rectangular field is $0.42 \mathrm{~nm}(0.031 \lambda)$. This compares favorably to the composite RMS wavefront of $0.28 \mathrm{~nm}(0.021 \lambda)$ with a transmission mask. The difference between the two imaging conditions is that the wavefront error varies more across the tilted conjugate planes. With a reflection mask, the wavefront error varies from $0.24 \mathrm{~nm}(0.018 \lambda)$ to $0.74 \mathrm{~nm}(0.055 \lambda)$. The wavefront error variation with a transmission mask is 0.15 $\mathrm{nm}(0.011 \lambda)$ to $0.36 \mathrm{~nm}(0.027 \lambda)$. While this variation across a tilted wafer would be troublesome in a production tool, causing field dependent $\mathrm{CD}$ variations across the field, it is not a significant issue for this R\&D tool 
Table 1.0 MET Projection Optics Performance Summary ( $\mathrm{RM}=$ Reflection Mask, $\mathrm{TM}=$ Transmission Mask)

\begin{tabular}{|c|c|}
\hline Parameter & Predicted Performance \\
\hline Wavelength & $13.4 \mathrm{~nm}$ \\
\hline Numerical aperture & 0.30 \\
\hline Focal length & $102.5 \mathrm{~mm}$ \\
\hline 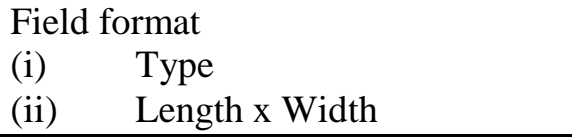 & $\begin{array}{c}\text { Rectangular } \\
600 \mu \mathrm{m} \times 200 \mu \mathrm{m} \\
\end{array}$ \\
\hline Mask Compatibility & RM \& TM \\
\hline Reduction ratio (nominal) & $5: 1$ \\
\hline $\begin{array}{l}\text { Residual RMS wavefront error } \\
\text { (waves @ } \lambda=13.4 \mathrm{~nm} \text { ) } \\
\text { (i) Field point maximum } \\
\text { (ii) Composite }\end{array}$ & $\begin{array}{l}0.054 \lambda(\mathrm{RM}) \\
0.027 \lambda(\mathrm{TM}) \\
0.031 \lambda(\mathrm{RM}) \\
0.021 \lambda(\mathrm{TM}) \\
\end{array}$ \\
\hline Resolution & $30 \mathrm{~nm}$ \\
\hline $\begin{array}{l}\text { Distortion (PTV static, nm) } \\
\text { (i) Chief ray }\end{array}$ & $\begin{array}{r}497.6 \mathrm{~nm}(\mathrm{RM}) \\
2.24 \mathrm{~nm}(\mathrm{TM}) \\
\end{array}$ \\
\hline Depth of focus & $200 \mathrm{~nm}$ \\
\hline $\begin{array}{l}\text { Telecentricity error } \\
\Delta \mathrm{y}(\mathrm{nm}) \text { image/ } \Delta \mathrm{z}(\mathrm{nm}) \text { focus }\end{array}$ & $0.0148 \mathrm{~nm} / \mathrm{nm}$ \\
\hline $\begin{array}{l}\text { Package } \\
\text { (i) } \\
\text { (ii) } \\
\text { (ii) }\end{array}$ & $\begin{array}{l}474.16 \mathrm{~mm} \\
275.60 \mathrm{~mm}\end{array}$ \\
\hline $\begin{array}{l}\text { Working distances } \\
\text { (i) M1/wafer } \\
\text { (ii) } \mathrm{M} 2 / \text { mask }\end{array}$ & $\begin{aligned} & \sim 5.0 \mathrm{~mm} \\
\sim & 113.56 \mathrm{~mm}\end{aligned}$ \\
\hline Aperture stop & Accessible on M1 \\
\hline $\begin{array}{l}\text { Peak aspheric departure } \\
\text { (i) } \\
\text { (ii) } \\
\text { (ii) (Primary) } \\
\text { M2 (Secondary) }\end{array}$ & $\begin{array}{l}3.82 \mu \mathrm{m} \\
5.61 \mu \mathrm{m}\end{array}$ \\
\hline $\begin{array}{l}\text { Maximum aspheric slope } \\
\text { (iii) } \\
\text { (iv) }\end{array}$ & $\begin{array}{l}-1.18 \mu \mathrm{m} / \mathrm{mm} \\
-0.47 \mu \mathrm{m} / \mathrm{mm}\end{array}$ \\
\hline $\begin{array}{l}\text { Angles of incidence } \\
\text { (i) } \quad \text { M1 (max/min) } \\
\text { (ii) } \quad \text { M2 (max/min) }\end{array}$ & $\begin{array}{l}8.67^{\circ} / 2.54^{\circ} \\
1.98^{\circ} / 0.67^{\circ}\end{array}$ \\
\hline
\end{tabular}




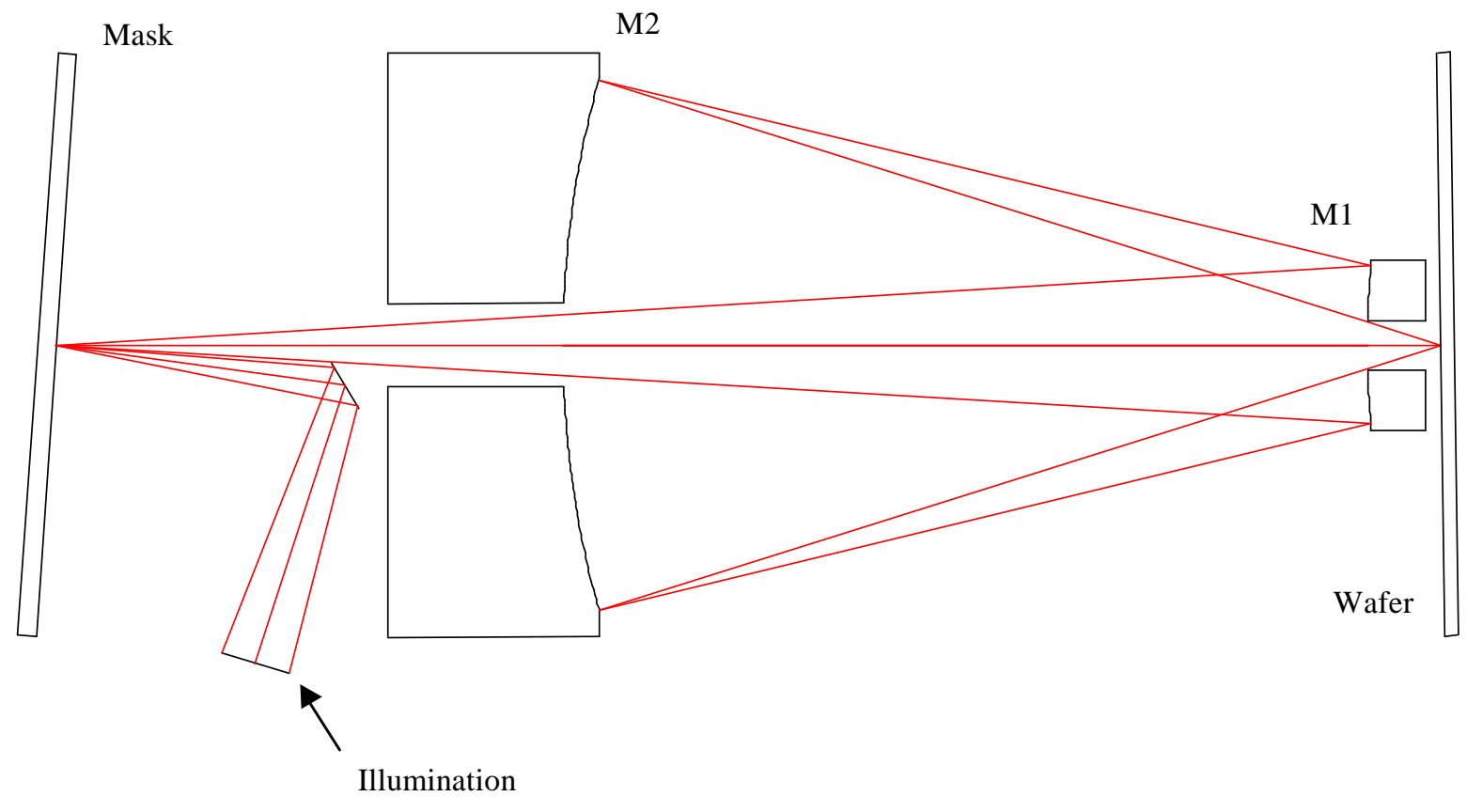

Figure 2. Tilting the mask and wafer planes enables use of a reflection mask. In this embodiment, the mask is tipped by $4.0^{\circ}$, with a corresponding wafer tilt of $0.8^{\circ}$. The imagery is diffraction-limited on tilted wafer plane.

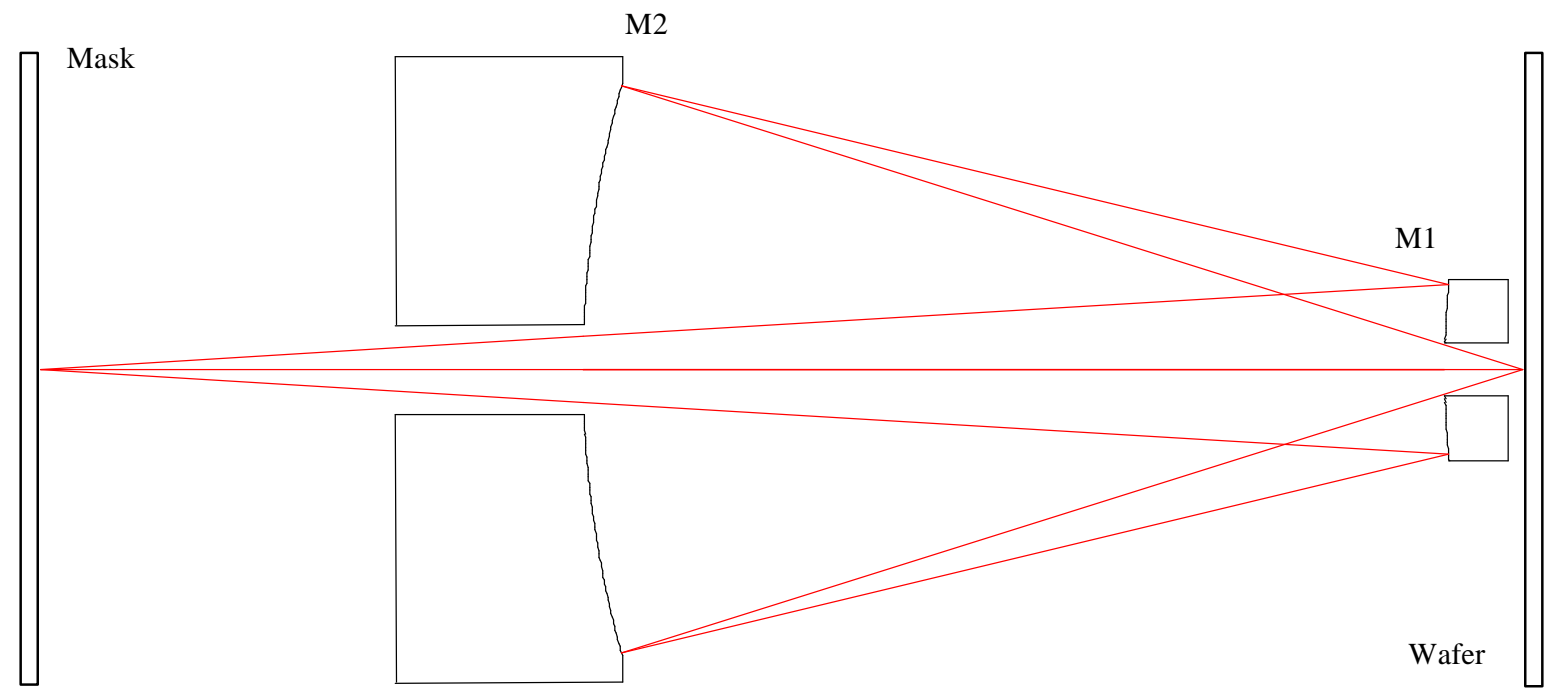

Figure 3. "Equal radii" microstepper concept for use with a transmission mask. The design has a numerical aperture of 0.30 at a reduction of $5 \mathrm{x}$ as measured at the plane of the wafer. Mirror radii R1 and R2 are nearly the same, acting to minimize field curvature across the projected format. 


\subsection{Detailed optical prescription}

A detailed optical design prescription in millimeters $(\mathrm{mm})$ including radii, thicknesses, and aspheric profile definitions is listed in Table 2.0. The table also includes relevant specification and aperture data needed to completely reconstruct the model in an optical design code.

The mask plane is denoted "OBJ" and is located $173.565 \mathrm{~mm}$ from the vertex of the secondary. Surface 1 is a dummy surface in the model used solely to determine the size of the hole required in the secondary. The aperture stop is colocated at the primary mirror, whose vertex radius is $318.13 \mathrm{~mm}$. This is also surface 2 , hence the designation of the primary "STO(2)". The larger secondary mirror has a

Table 2.0 MET optical design prescription (reflection mask)

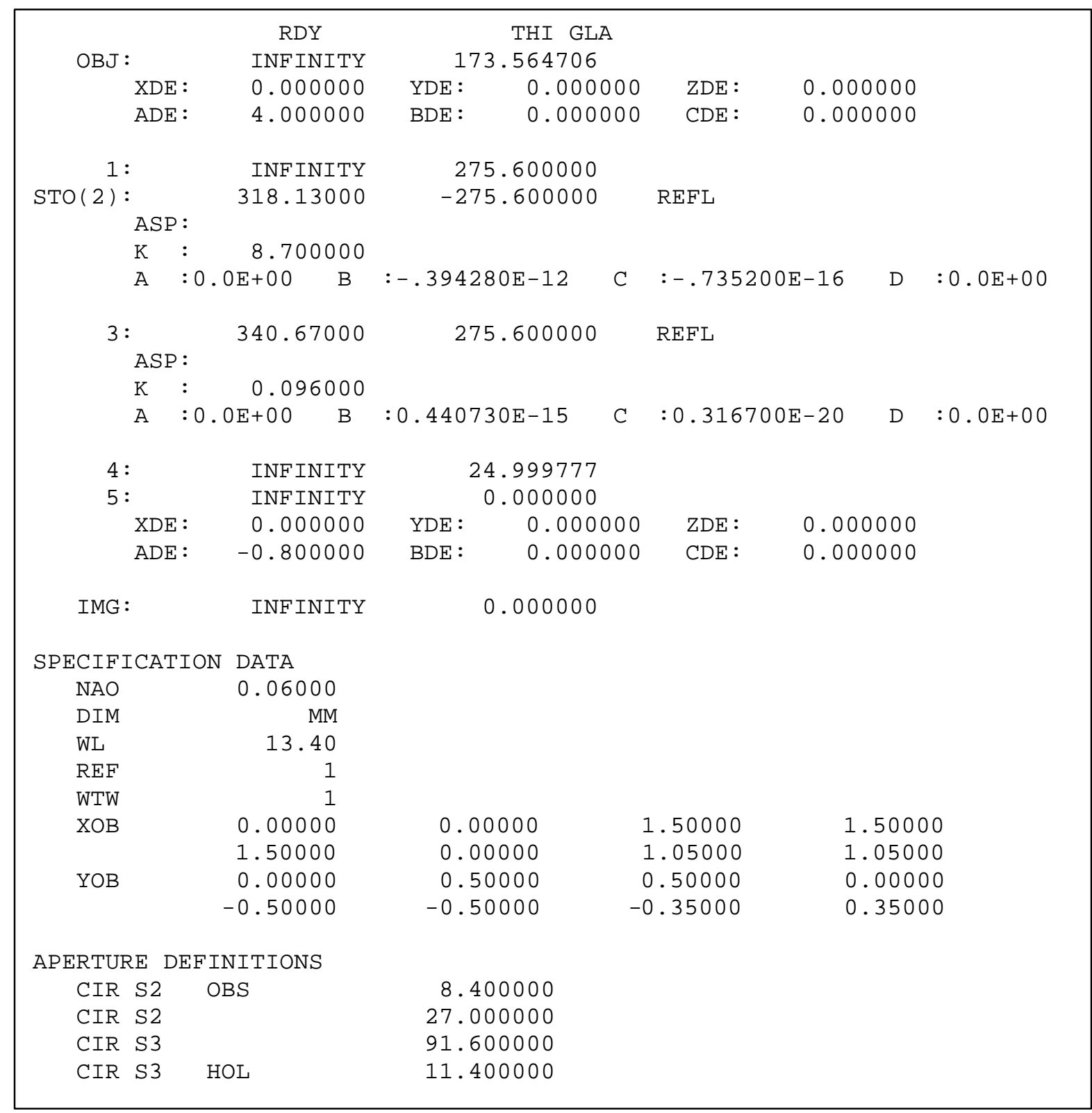


vertex radius of $340.67 \mathrm{~mm}$ and is designated " 3 ". Surface " 4 " is another dummy surface located at the vertex of the primary, again used to size the required clearance hole in this mirror. The distance from the vertex of the primary and the wafer along the optical axis is $25 \mathrm{~mm}$ (thickness of surface " 4 "). The clearance at the wafer is not readily discerned from this listing since it depends on the thickness of the primary substrate, which is actually $20 \mathrm{~mm}$. Therefore, the clearance between the back of the primary and the wafer is $5 \mathrm{~mm}$ along the optical axis. Due to the tilt of the wafer plane and the final diameter of the substrate (which will be larger than what is listed here), the actual minimum clearance will be somewhat less than $5 \mathrm{~mm}$.

With the mask plane tilted $4.0^{\circ}$, the corresponding wafer plane tilt is $-0.8^{\circ}$. The wafer plane tilt is added via a dummy surface (surface "S5") that preceeds the wafer . Both tilts have an "ADE" designation. The model of the MET using a transmission mask is simple created by zeroing the tilts on the object surface and the dummy surface.

Specification data includes the wavelength of use $(13.4 \mathrm{~nm})$, the numerical aperture at the mask (0.06) and the object field points in $\mathrm{x}$-dimension and y-dimension at the mask. Aperture definitions are based on a raytrace footprint analysis using the $3.0 \mathrm{~mm} \times 1.0 \mathrm{~mm}$ rectangular field of view at the mask. These aperture definitions are flexible in that they may be refined to include bevels and "keep-out" zones to accommodate the manufacturing and multilayer coating process. The strategy is to keep the outer radii of the clear aperture for the primary $(27.0 \mathrm{~mm})$ and secondary $(91.6 \mathrm{~mm})$ fixed, but allow the inner radii of the holes in the primary $(8.4 \mathrm{~mm})$ and secondary $(11.4 \mathrm{~mm})$ to be refined $(\sim 0.5 \mathrm{~mm})$ based on fabrication input.

To achieve imagery corrected to lithographic standards, the primary and secondary make use of aspheric surfaces to correct the residual aberrations. The base aspheres are ellipsoids with $6^{\text {th }}$ and $8^{\text {th }}$ order polynomial deformations described by the $\mathrm{B}$ and $\mathrm{C}$ coefficients, respectively. In this design the conic constants $(\mathrm{K})$ are greater than 1.0, indicating that both mirrors are oblate spheroids (sections an ellipse rotated about the minor axis). The principle aspheric departure is controlled by the base conic, however, the higher-order deformations are significant since they provide several additional waves of aberration correction across the large numerical aperture. Plots of the aspheric departure relative to the best fit sphere across the clear aperture are shown for the primary and secondary in Figure 4 and Figure 5, respectively. The radius of the best fit sphere is computed as the radius that minimizes the peak aspheric departure, while satisfying the constraint that the sphere contacts the aspheric surface at the edges of the clear aperture (an area-weighted fit is not used!). For the MET primary, the radius of the best fit sphere is $312.625 \mathrm{~mm}$ (computed over the clear aperture defined from $8.4 \mathrm{~mm} \leq \mathrm{h} \leq 27.0 \mathrm{~mm}$ ). For the MET secondary, the radius of the best fit sphere is $340.0541 \mathrm{~mm}$ (computed over the clear aperture defined from $11.4 \mathrm{~mm} \leq \mathrm{h} \leq 91.6 \mathrm{~mm}$ ).

Final convergence on the MET design was also driven by auxiliary considerations. First, the base radii of the primary and secondary were chosen specifically to match, as closely as possible, the radius of the M3 mirror of the ETS optical system $\left(\mathrm{R}_{\mathrm{M} 3}=388.25 \mathrm{~mm}\right)$. Since, like $\mathrm{M} 3$, the MET primary and secondary are rotationally symmetric, much of the existing M3 PSDI test set (laser, delay lines, phase shifter, software, etc.) can be used without modification. This philosophy has the potential to reduce the cost of testing specific components including the transmission sphere, imaging objective, and fixturing.

Secondly, the aspheric departure was carefully tailored in such a way to provide a development path for potential EUVL projection optics suppliers. The diameter of the secondary $(\sim 183 \mathrm{~mm})$, peak aspheric departure $(5.61 \mu \mathrm{m})$, and aspheric surface slope $(\sim 0.5 \mu \mathrm{m} / \mathrm{mm})$ classify this optic as an "ETS-type" mirror. 


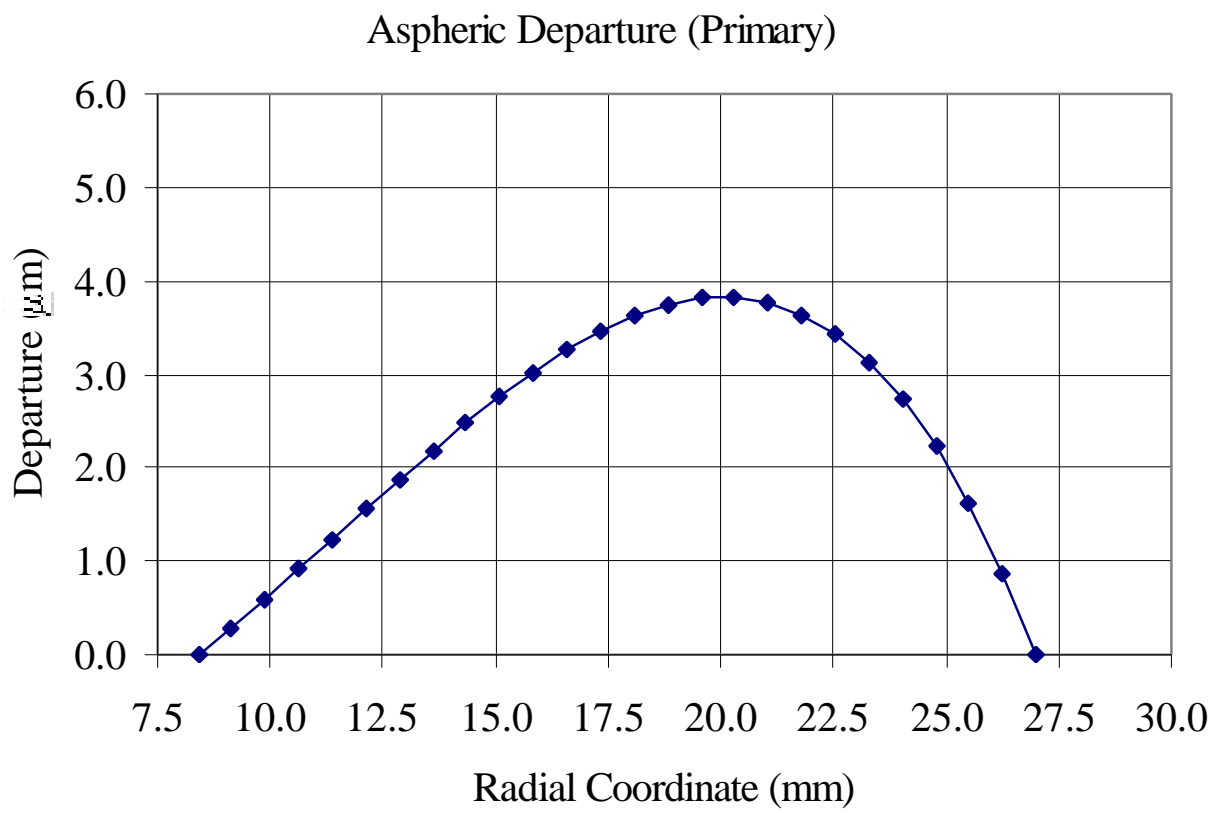

Figure 4. Aspheric departure of primary mirror from best-fit sphere $\left(\mathrm{R}_{\mathrm{BFS}}=312.625 \mathrm{~mm}\right)$. The peak aspheric departure is $3.82 \mu \mathrm{m}$, and the maximum slope is $-1.18 \mu \mathrm{m} / \mathrm{mm}$. While the peak aspheric departure is well-controlled, this mirror is challenging since the aspheric slope near the edge of the clear aperture is $\sim 40 \%$ greater than that of the ETS mirrors.

Aspheric Departure (Secondary)

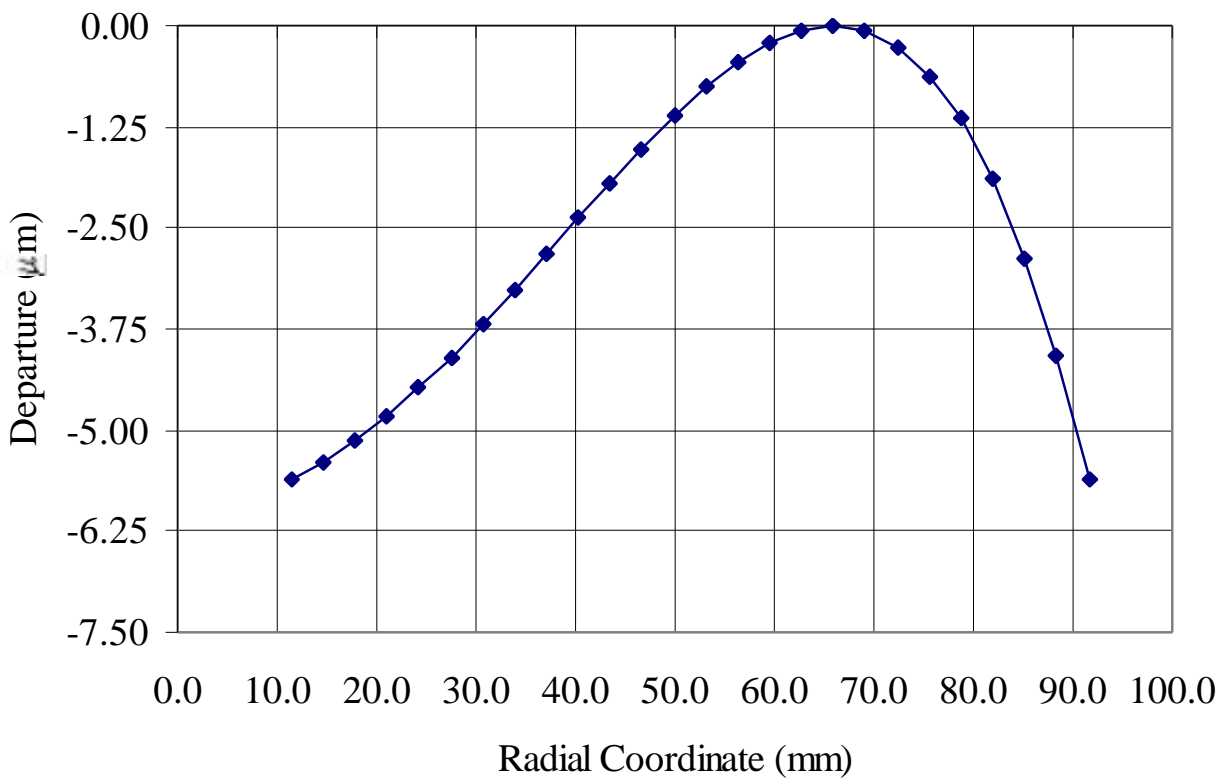

Figure 5. Aspheric departure of secondary mirror from best-fit sphere $\left(\mathrm{R}_{\mathrm{BFS}}=340.051 \mathrm{~mm}\right)$. The peak aspheric departure is $-5.61 \mu \mathrm{m}$, and the maximum slope is $-0.47 \mu \mathrm{m} / \mathrm{mm}$. Both the peak aspheric departure and slope is well-controlled, making the fabrication effort similar to that of the ETS mirrors. 


\subsection{Detailed wavefront and distortion analysis on the tilted plane}

Since the MET camera is compatible with either a reflection mask (RM) or a transmission mask (TM), the wavefront error and distortion analysis is performed in both modes of operation. The RMS wavefront error was analyzed at 9 distinct field point across the half format as shown in Figure 6. This sampling is sufficient since the design has bilateral symmetry. The field size is set in RM mode by the requirement that the maximum RMS wavefront error at all field points is less than 0.050 $\lambda$. The RMS wavefront error, less tilt, for each field point is listed in Table 3.0. Using a transmission mask, the field composite RMS wavefront error is $0.28 \mathrm{~nm}(0.021 \lambda)$. Residual field curvature and astigmatism present in the design cause a slight variation in the residual wavefront error across the field. With a reflection mask, the field composite RMS wavefront is $0.42 \mathrm{~nm}(0.031 \lambda)$. There is more variation in the wavefront error in this case. This is due primarily to a variation in spherical aberration (fringe Zernike term Z9) across the field. This is a subtle effect that can be understood in the following way. With a tilted mask plane, the distance from the object surface to the first principal plane varies across the field, creating a field-dependent conjugate shift. Since the spherical aberration varies with conjugate distance, the spherical aberration will now have field dependence.

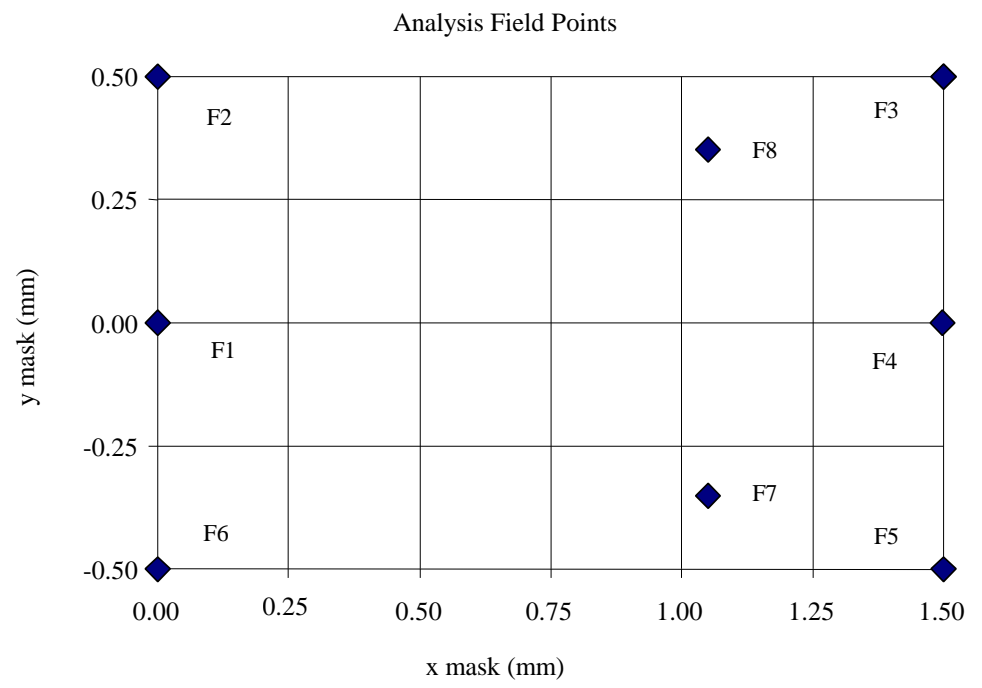

Figure 6. Analysis field points for the computation of RMS wavefront error, incoherent square wave MTF, and 2D/3D partially coherent imagery. With a reflection mask, the printed field has bilateral symmetry, thus only field points across the halfformat need be analyzed.

Table 3.0 RMS Wavefront Error (WFE) ${ }^{1}$

\begin{tabular}{|c|c|c|c|c|}
\hline Field & $\mathrm{x}(\mathrm{mm})$ & $\mathrm{y}(\mathrm{mm})$ & $\begin{array}{c}\text { RMS WFE } \\
\text { (Transmission Mask) }\end{array}$ & $\begin{array}{c}\text { RMS WFE } \\
\text { (Reflection Mask) }\end{array}$ \\
\hline F1 & 0.00 & 0.00 & $0.022 \lambda$ & $0.022 \lambda$ \\
\hline F2 & 0.00 & 0.50 & $0.018 \lambda$ & $0.046 \lambda$ \\
\hline F3 & 1.50 & 0.50 & $0.027 \lambda$ & $0.020 \lambda$ \\
\hline F4 & 1.50 & 0.00 & $0.022 \lambda$ & $0.024 \lambda$ \\
\hline F5 & 1.50 & -0.50 & $0.027 \lambda$ & $0.054 \lambda$ \\
\hline F6 & 0.00 & -0.50 & $0.018 \lambda$ & $0.018 \lambda$ \\
\hline F7 & 1.05 & -0.35 & $0.011 \lambda$ & $0.024 \lambda$ \\
\hline F8 & 1.05 & 0.35 & $0.011 \lambda$ & $0.023 \lambda$ \\
\hline & & Composite & $0.021 \lambda$ & $0.031 \lambda$ \\
\cline { 3 - 5 }
\end{tabular}

${ }^{1}$ Tilt removed 


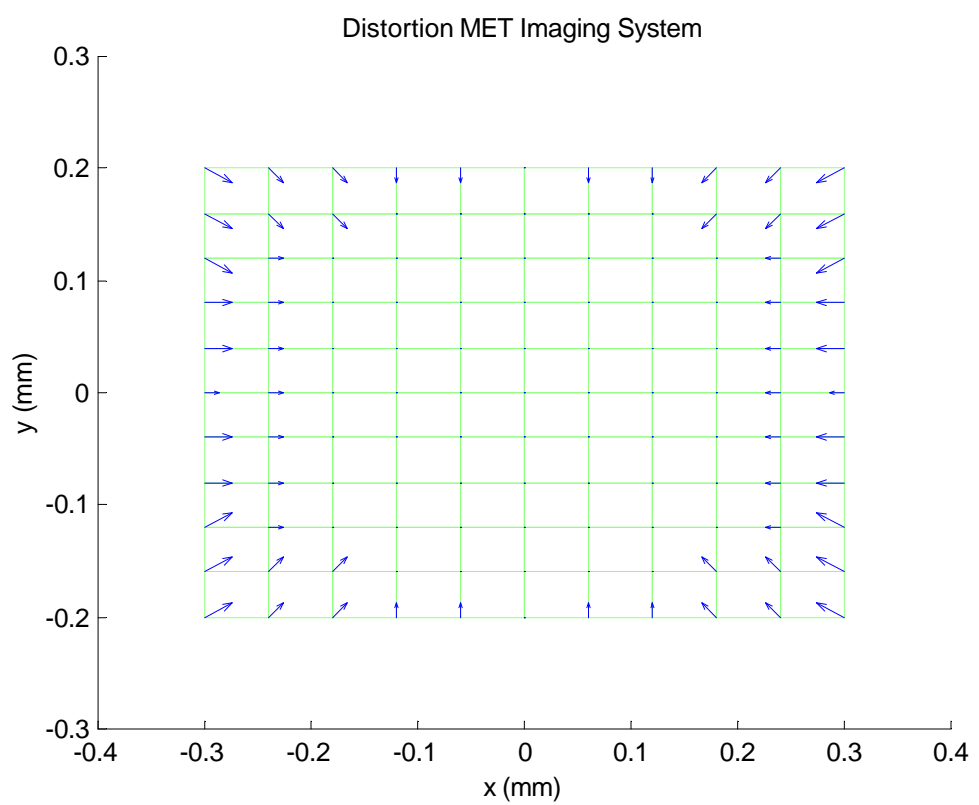

Figure 7. Vector visualization of MET distortion using a transmission mask over a $600 \mu \mathrm{m}$ x $200 \mu \mathrm{m}$ imaging field at the wafer. The maximum radial distortion is only $2.24 \mathrm{~nm}$. Since the system is rotationally symmetric in this mode of operation, the distortion field has rotational symmetry about the optical axis.

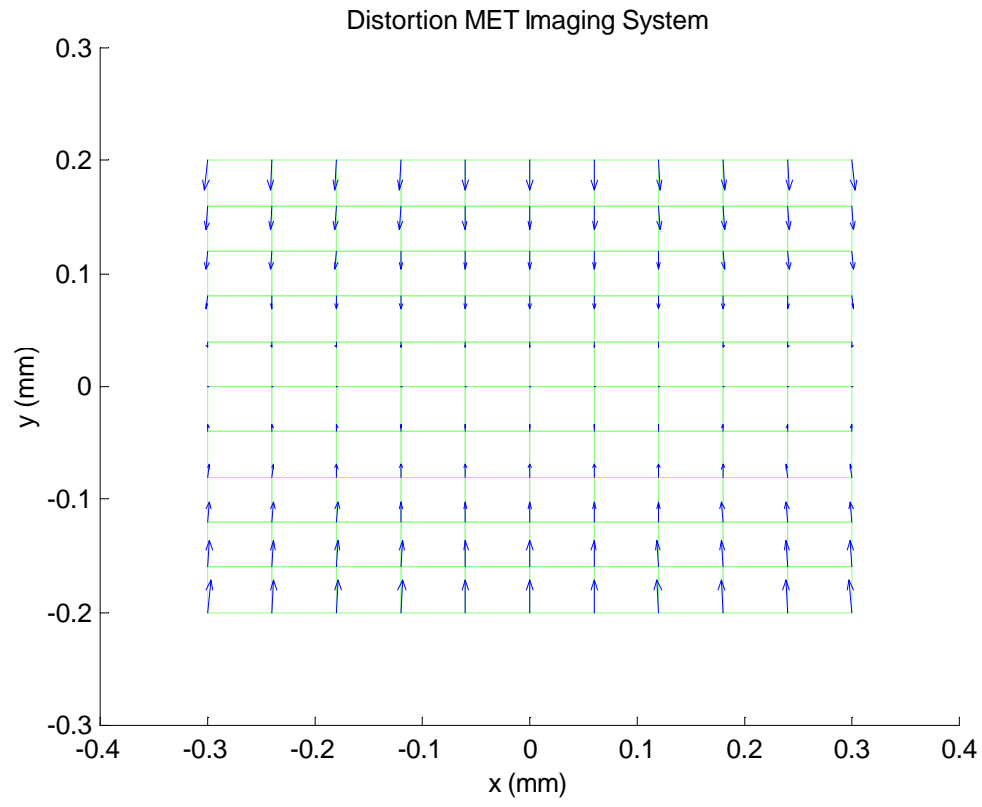

Figure 8. Vector visualization of MET distortion using a reflection mask over a $600 \mu \mathrm{m}$ x $200 \mu \mathrm{m}$ imaging field at the wafer. In this case, the maximum radial distortion is $497.9 \mathrm{~nm}$. The printed image suffers primarily from anamorphic distortion, which can be viewed simple as a foreshortening of the vertical dimension. Keystone distortion can also be seen in the vector field plot. Both forms of distortion are artifacts of imaging using the Scheimpflug condition. 
Figure 7 and Figure 8 graphically depict the distortion at the wafer with a transmission mask and reflection mask, respectively. The distortion vector field is superimposed on top of the ideal image grid. Even though there is no overlay requirement for this research tool, there is still need to understand the distortion fields in both imaging modes to be able to address such issues as horizontal/vertical bias with tilted plane imaging and the potential to use this design in a scanning configuration.

With the transmission mask being perpendicular to the optical axis, the distortion field exhibits simple barrel distortion with rotational symmetry about this axis (Figure 7). The length of the largest distortion vector (and hence the maximum radial distortion) is $2.24 \mathrm{~nm}$, with maxima being located in the corners of the format. There are no degrees of freedom in the optical design to correct distortion effectively; the distortion is minimized only because the projected field of view is small. An analysis of the scanned imagery shows an image placement error of $\sim 2 \mathrm{~nm}$ in the cross-scan dimension. The residual distortion is small enough to consider the possibility of using this design with a transmission mask in a scanning configuration.

Behavior of the distortion field is much more complex using a reflection mask (Figure 10). The printed image suffers primarily from anamorphic distortion, which can be viewed as a foreshortening of the vertical dimension due to the tilted plane. Keystone distortion can also be seen in the vector field plot, which relates to the variation in magnification with conjugate distance from the mask to the first principle plane. Both forms of distortion are artifacts of imaging using tilted conjugate planes. The longest distortion vector in the field plot is $\sim 498 \mathrm{~nm}$ in length. Barrel distortion is also present, but is overwhelmed by the other distortion forms. The conclusion of this analysis is that the MET camera is not suited for use in a scanning tool using a reflection mask.

\subsection{Incoherent square wave modulation transfer function (MTF)}

By studying the incoherent square wave response at spatial frequencies out the desired resolution, the performance of the optical design can be assessed quickly without the need for a lengthy study of the partially coherent imagery. A good "rule of thumb" is that the optical design should have an incoherent MTF that matches the diffraction limit with a contrast greater than 0.4 at the desired resolution limit. The incoherent square wave response for the MET camera with a reflection mask illustrated in Figures 9 and 10. At a numerical aperture of 0.30 , the design achieves near diffraction-limited performance to 16,667 $\mathrm{lp} / \mathrm{mm}$ (corresponding to $30 \mathrm{~nm} \mathrm{~L} / \mathrm{S}$ ). The slight variation in contrast is a result of the variation in RMS wavefront error across the field, which indicates that there will be some measure of CD non-uniformity across the field. However, this CD variation should be of little consequence given the low aberration residuals and research nature of the tool.

The through-focus MTF at 16,667 lp/mm (30 nm L/S) is shown in Figure 10. These MTF curves depict how the contrast varies with focus position for selected points in the field of view. The response for each target orientation is essentially the same through-focus, indicating the lack of an astigmatic or comatic residual. The peaks of the contrast curve do not precisely overlap, indicating a small level of field curvature still exists in the design. The depth of focus may be estimated by accepting that the DOF is defined by a loss in contrast of 0.1 . Using this metric, Figure 10 demonstrates that the design is capable of supporting a depth-of-focus of $\sim 0.2 \mu \mathrm{m}$ for a feature size of $30 \mathrm{~nm}$. 


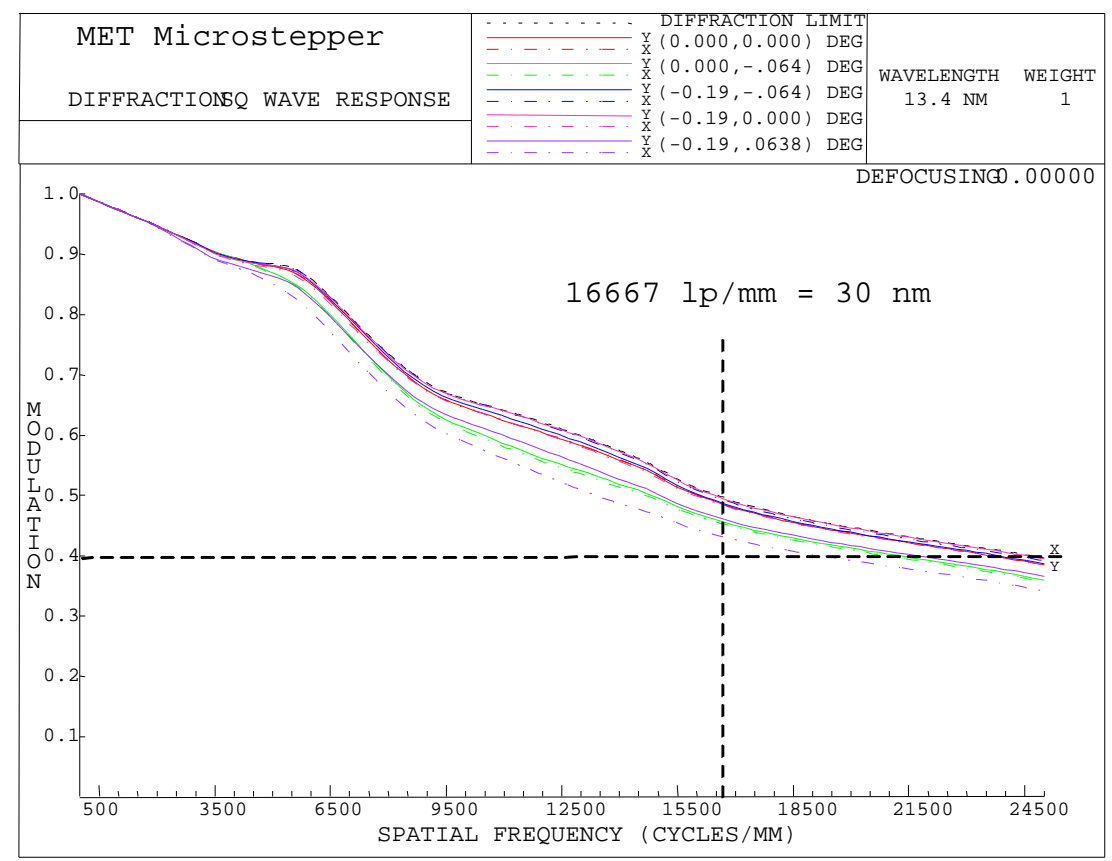

Figure 9. Incoherent square wave response for selected field points across $600 \mathrm{~mm}$ x $200 \mathrm{~mm}$ field exhibits resolution well past $30 \mathrm{~nm}(16,667 \mathrm{lp} / \mathrm{mm})$

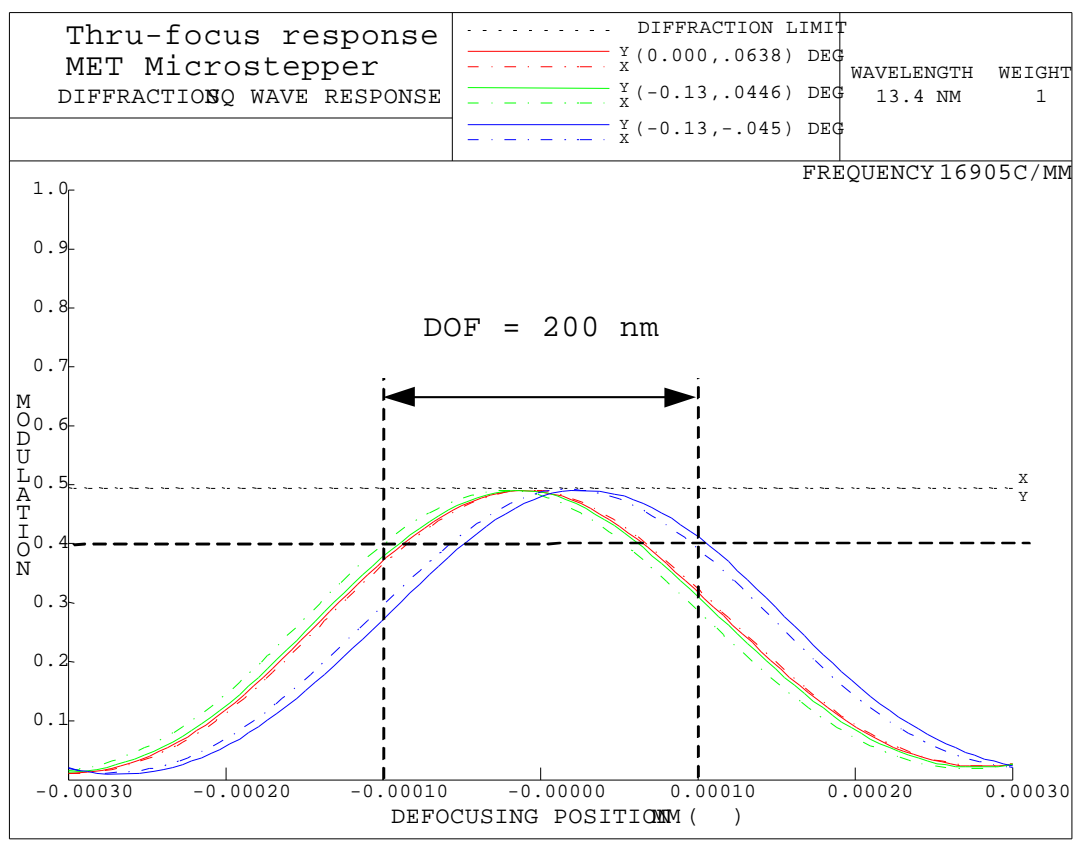

Figure 10. Through focus MTF illustrates a depth of focus that is $\sim 200 \mathrm{~nm}$. There is little difference in contrast between the tangential and sagittal orientations, indicating that coma and astigmatism are well corrected. The variation in peak response for different field points is a result of the residual field curvature. 


\subsection{Initial assessment of partial coherent imaging characteristics}

For a lithographic imaging system, it is insufficient to analyze performance only in terms of RMS wavefront error and incoherent MTF. First, the imaging is partially coherent and the pupil fill geometry has a dramatic impact on image formation. Second, the square wave MTF is only valid for equal lines and spaces and tells us nothing about how isolated lines or isolated contact holes print. Lastly, traditional optical metrics are not directly related to lithographic quality, so the estimation of performance is only approximate at best.

Our initial assessment of lithographic performance included a computation of the focus-exposure matrix (FEM) and corresponding process window using Prolith3D. The simulations used a threshold resist model, a partial coherence $(\sigma)$ of 0.7 , and included the residual aberrations of the optical design. Figure 11 shows the FEM and corresponding focus-exposure process window. Although other items such as sidewall angle and resist loss are depicted in the figure, the process window is determined solely by a $\pm 10 \%$ change in CD at the nominal feature size of $30 \mathrm{~nm}$. The process window can therefore be

Resist Feature Width (nm)

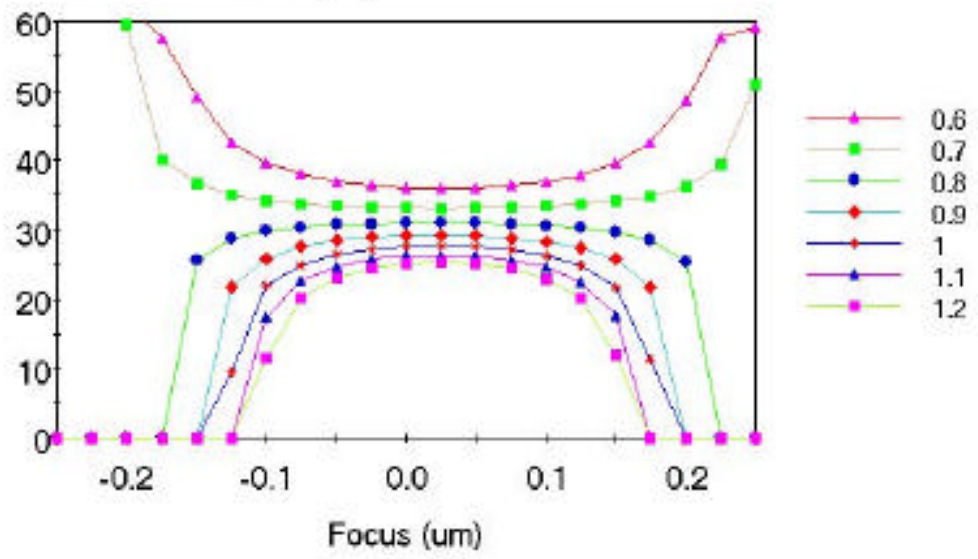

Exposure Delta (\%)

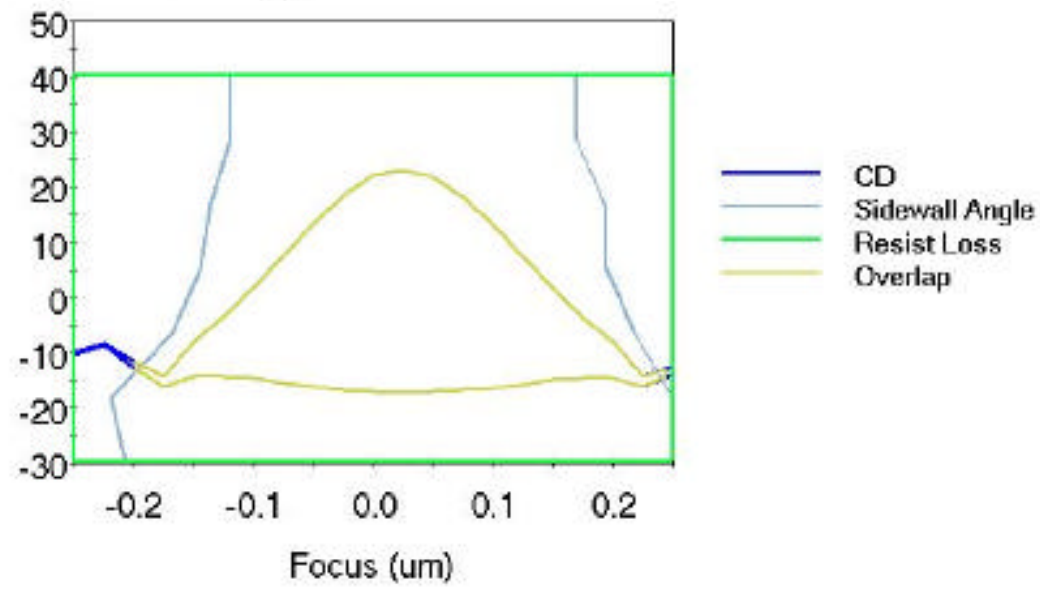

Figure 11. Focus exposure matrix (FEM) and corresponding CD process window for MET microstepper. The FEM and corresponding CD process window are calculated assuming a partial coherence $(\sigma)$ of 0.7 using the residual aberrations of the optical design. The effect of the central obscuration is also included. Assuming a exposure variation and $\mathrm{CD}$ variation of $\pm 10 \%$, the depth of focus is $\sim 0.2 \mu \mathrm{m}$. At best focus, the design can tolerate an exposure variation of $+20 \% /-15 \%$ while still maintaining an acceptable change in $\mathrm{CD}$. 


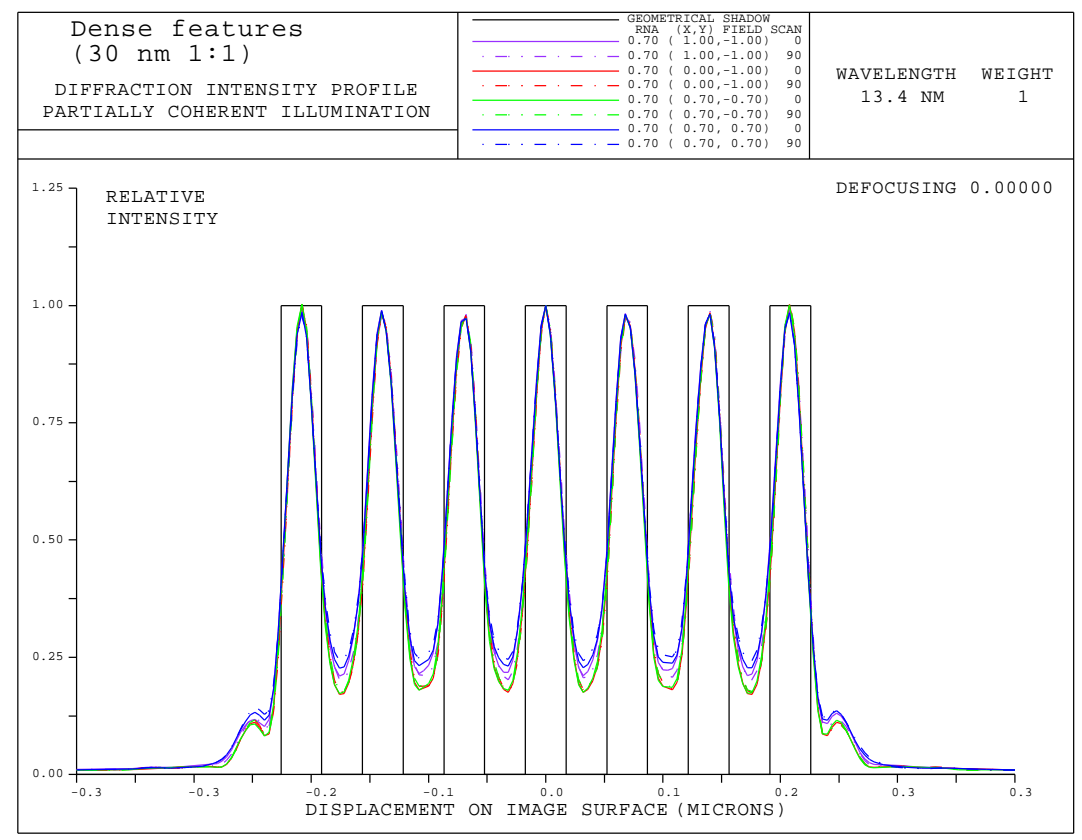

Figure 12. Two-dimensional partially coherent aerial images $(\sigma=0.7)$ of dense 1:1 $30 \mathrm{~nm}$ lines and spaces (L/S). Aerial images demonstrate $30 \mathrm{~nm}$ resolution with good $\mathrm{CD}$ uniformity across the printed field.

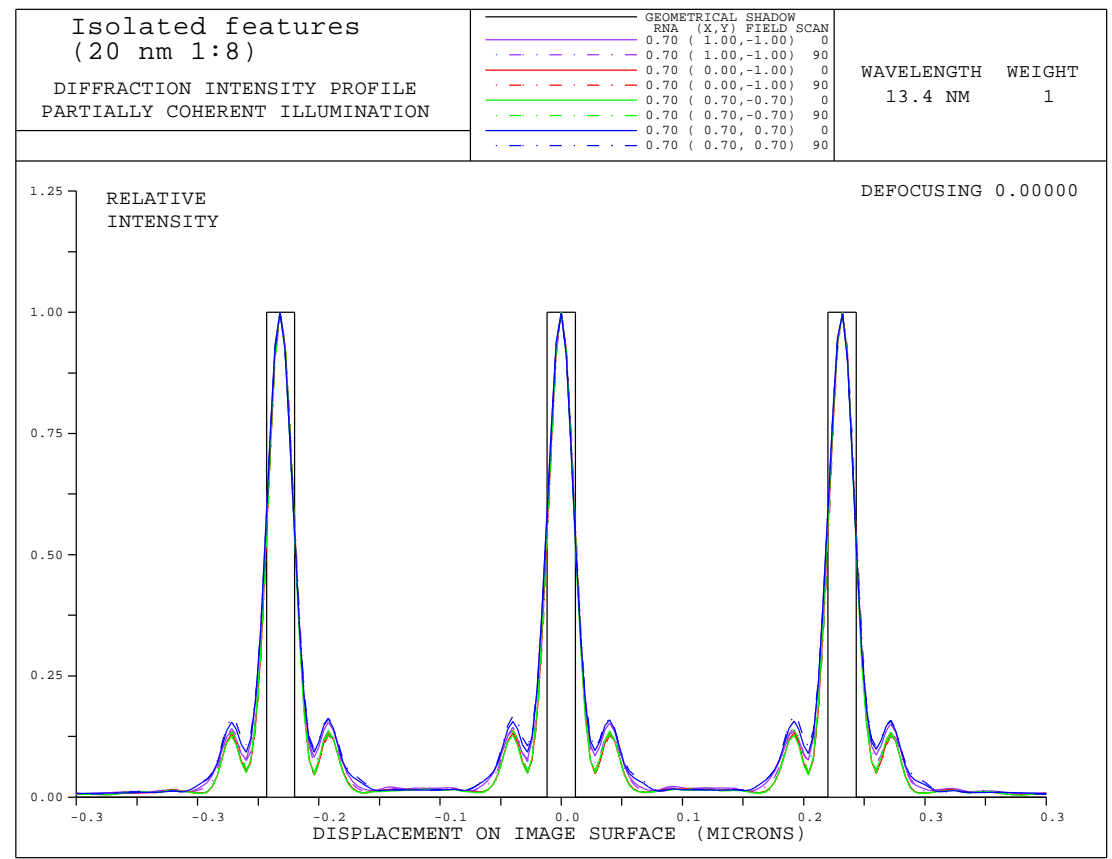

Figure 13. One-dimensional partially coherent imagery of isolated 1:8 $20 \mathrm{~nm}$ lines. Superimposed aerial images demonstrate $20 \mathrm{~nm}$ resolution of isolated features with good CD uniformity across the printed field. 
considered a $\mathrm{CD}$ process window and used to determine the depth of focus rigorously. Assuming that a variation in exposure and $\mathrm{CD}$ of $\pm 10 \%$ defines the process window, the allowable change in focus from the nominal focus setting is $\sim 0.2 \mu \mathrm{m}$. At best focus, the design can tolerate an exposure variation of $+20 \% /-15 \%$, within the limits of a $\pm 10 \% \mathrm{CD}$ variation. This large exposure variation at the nominal focus can be attributed to the steep log-slope of the aerial images at this focus. As expected, the CD process window narrows as the departure from nominal focus grows, making the system more sensitive to variations in exposure.

Two-dimensional partially coherent $(\sigma=0.7)$ aerial images of both dense and isolated features were also computed and are shown in Figure 12 and Figure 13, respectively. Normalized aerial images from across the field of view have been overlaid and plotted against ideal geometry image, allowing a visual estimate of CD uniformity at best focus. The effect of the central obscuration is immediately seen in the broadening of the wings of the aerial images. However, a simple threshold analysis of the figure demonstrates that the variation in CD across the field is conservatively less than $5.0 \%$ of the ideal geometric feature (1/20 of the linewidth), so an initial bound on the CD uniformity across the field is \pm $2.5 \%$. This conclusion holds for both dense and isolated features, indicating that both features types will print at the resolution limit with a good measure of CD uniformity for a research tool. More detailed image processing is currently being performed to extract more quantitatively how the $\mathrm{CD}$ varies across the field. This analysis will be presented in a subsequent report.

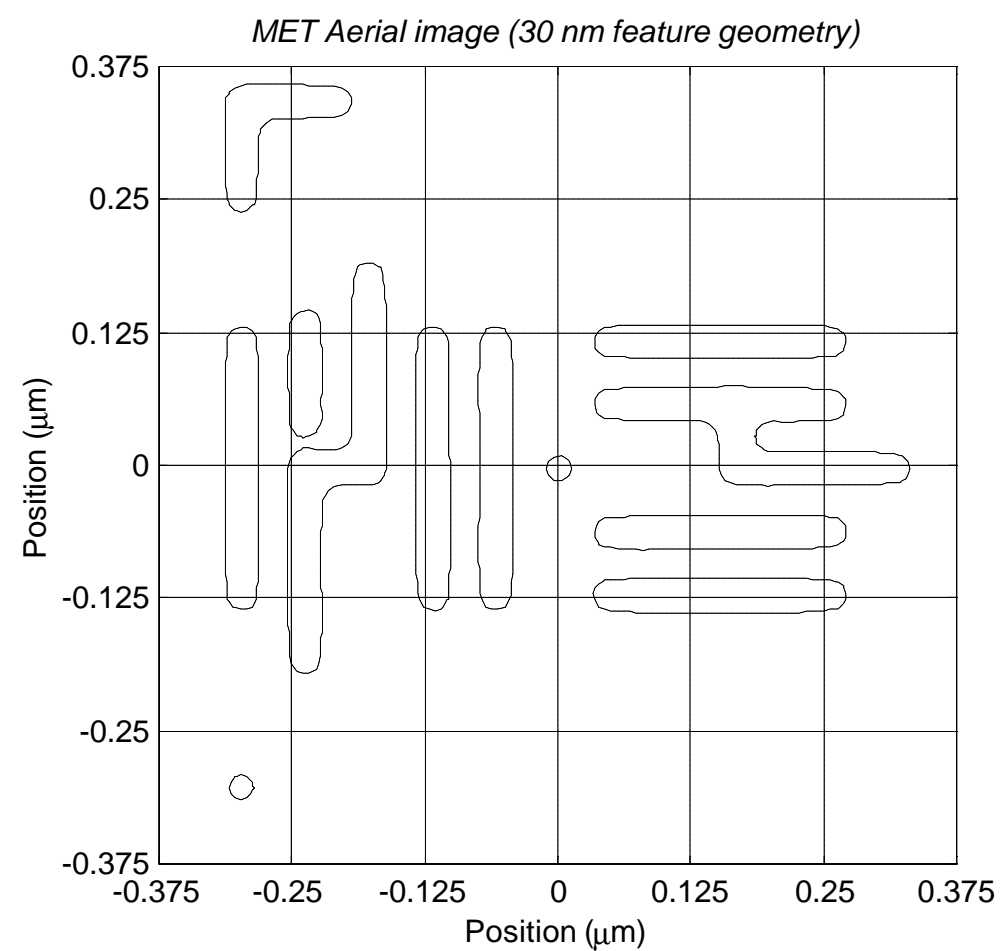

Figure 14. Threshold of a 3D partially coherent image of $30 \mathrm{~nm}$ test geometry consisting various dense and isolated features. The threshold was set to give a $30 \mathrm{~nm}$ linewidth for dense 1:1 L/S. This image demonstrates capability of the optical system to image a variety of features at the ultimate resolution limit of $30 \mathrm{~nm}$.

It is not enough to study partially coherent images of simple dense lines and spaces. A more comprehensive study will include 3D simulations of more complex mask geometry, the intent being to understand the fundamental limits to printing with the optical system. One such simulation is shown in 
Figure 14, which is a threshold of a 3D aerial image set at the nominal $30 \mathrm{~nm}$ linewidth for dense 1:1 L/S. This particular geometry consists of a variety of dense features, isolated features, elbows, and contacts. These initial results are promising and demonstrate, via existence proof, that there are no fundamental limitations to the types of geometry that can be studied with the MET optical system.

\subsection{Conclusion}

This report provides comprehensive documentation of the optical design that supports International Sematech's High NA Optics Development Project. This simple high NA optical system is designed for a microstepper and makes use of the "equal radii" concept to correct the field curvature over a $600 \mu \mathrm{m} \mathrm{x}$ $200 \mu \mathrm{m}$ field at the wafer. The projection system is designed to work at a reduction ratio of 5:1. Two aspheric mirrors are used in a coaxial, obscured configuration to achieve the high numerical aperture (NA) of 0.30 . The area obscuration is carefully limited to less than $10 \%$ of the exit pupil area, allowing the optical system to achieve sub $30 \mathrm{~nm}$ resolution with partially coherent illumination. Printing experiments can be performed either with a reflection or transmission mask, enabling two distinct modes of operation. To use a reflection mask, the mask plane is itself is tilted to allow the illumination to enter the projection optics. There is a corresponding tilt to the wafer plane that allows the design to recover most of its nominal performance. Diffraction-limited performance across the image field is achieved in either imaging mode. Using a transmission mask, the field composite RMS wavefront error is $0.28 \mathrm{~nm}$ $(0.021 \lambda, \lambda=13.4 \mathrm{~nm})$. With a reflection mask, the field composite RMS wavefront is $0.42 \mathrm{~nm}(0.031 \lambda)$.

Distortion with a transmission mask is small, controlled to less than $2.25 \mathrm{~nm}$ in the corners of the rectangular field. This residual distortion is small enough to consider using this projection system in a scanning, rather than stepping, architecture. The magnitude of the distortion grows rapidly with a reflective mask since it must be tilted $\left(4.0^{\circ}\right)$. Anamorphic and keystone distortion dominate throughout the field, with maximum radial error in image placement of $498 \mathrm{~nm}$. This discounts the concept of using the MET camera in a scanning tool based on a reflective mask architecture.

In addition to analysis using traditional optical metrics, an initial analysis of the partially coherent imagery was also performed. Specifically, a focus-exposure matrix (FEM) matrix and corresponding CD process window were generated. With an exposure variation and CD variation of $\pm 10 \%$, the depth of focus was found to be $\sim 0.2 \mu \mathrm{m}$. At best focus, the design can tolerate an exposure variation of $+20 \% /-$ $15 \%$, within the limits of a $\pm 10 \% \mathrm{CD}$ variation. The CD uniformity across the field for both dense and isolated features was estimated conservatively from $2 \mathrm{D}$ aerial image profiles to be better than $\pm 2.5 \%$ for $30 \mathrm{~nm}$ features, which is adequate for a research tool. Three-dimensional partially coherent aerial images of a sample test geometry containing dense features, isolated features, isolated elbows and contacts demonstrate, via existence proof, that the MET optical system will print a variety of features to the ultimate resolution limit of $30 \mathrm{~nm}$ without limitation.

\subsection{Auspices}

This work was performed under the auspices of the U.S. Department of Energy by the Lawrence Livermore National Laboratory under Contract No. W-7405-ENG-48. Funding was provided by the Extreme Ultraviolet Limited Liability Corporation under a Cooperative Research and Development Agreement. This effort is in support of International Sematech Project LITH112 -- High-NA EUV Optics for Mask Defect Printability Scaling under the administration of Neil Wester. 


\section{Appendix A}

R. Hudyma, "Projection Concepts for Advanced Microstepper," AMP Technical Memorandum M9801124, August 31, 1998. 


\section{Advanced Microtechnology Program}

August 31, 1998

To: Distribution

From: Russ Hudyma

Subject: Projection concepts for advanced microsteppers

\subsection{Introduction}

This memorandum describes high numerical aperture optical designs $(0.13-0.25)$ for an advanced microstepper. The designs offer higher resolution over fields that are either the same or larger than the current 10x projection system. These designs could be used to enable sub-70 $\mathrm{nm}$ printing experiments substantially before first light is reached on the ETS.

Two design ideas are described in which aspheric mirrors are used to increase the numerical aperture. It is shown that transmission masks enable numerical apertures up to 0.25 , while numerical apertures on the order of 0.13 are possible using reflection masks. One design is derived from the basic Schwarzschild configuration; the other uses the idea of "equal radii" to overcome the residual field curvature of the Schwarzschild. The latter design has $1.5 \mathrm{~mm}$ x $0.5 \mathrm{~mm}$ rectangular field at the wafer which is an order of magnitude larger than that of the current 10x camera. For consistency, each system is designed to work at $5 \mathrm{x}$ reduction ${ }^{1}$ with an effective focal length of $100 \mathrm{~mm}$. Although there are additional issues to investigate, the "equal radii" concept is shown to be superior. This memorandum briefly documents each concept and provides a quantitative comparison of the static imaging performance using traditional optical metrics.

\subsection{Fundamental field of view limitations}

Every optical system has an intrinsic field curvature associated with the layout of the design that is called the Petzval or field curvature. A practical limit on field curvature can be set using the depth of focus determined from Rayleigh's quarter wave criterion. Assuming that the field must stay flat to within the depth of focus as defined by Rayleigh, it can be shown that the maximum semi-field of view ( $\mathrm{y}_{\text {semi }}$ ) for a 2-mirror system is given by

$$
y_{\text {semi }}=\sqrt{\frac{\lambda}{2 N A^{2}}\left(\frac{R_{1} R_{2}}{R_{2}-R_{1}}\right)},
$$

where $\lambda$ is the wavelength, NA is the numerical aperture, and $R_{1}$ and $R_{2}$ are the mirror radii of the primary and secondary mirrors, respectively. The full linear field of view is simply $2 \mathrm{y}_{\text {semi }}$. For a classic Schwarzschild, the mirror radii are related to the focal length via $R_{1}=(\sqrt{5}-1) f$ and $R_{2}=(\sqrt{ } 5+1) f$. Substituting these values into the expression for the maximum semi-field yields,

$$
y_{\text {semi }}=\sqrt{\frac{\lambda}{N A^{2}} \mathrm{f}} .
$$

\footnotetext{
${ }^{1}$ Printable mask defect experiments require a 4-5x reduction; the $5 \mathrm{x}$ reduction provides the best ray clearance.
} 
The maximum semi-field is related to both the focal length of the objective and its numerical aperture. As the NA is increased, the depth of focus is decreased, thus limiting the amount of field curvature that can be tolerated. The only recourse in this instance is to increase the focal length of the objective for a given NA.

Figure 1. Field of view vs. NA trade space for Schwarzschild objectives. The maximum semifield is plotted as a function of NA for several practical focal lengths. As the focal length is scaled up to achieve semi-fields of $500 \mu \mathrm{m}$ (corresponding to a full field of $1 \mathrm{~mm})$, the mirrors will become large. For reference, the 10x camera lies on the lower left on the graph. The inability to correct the Petzval sum represents the fundamental limitation to the development of a wide field microstepper.

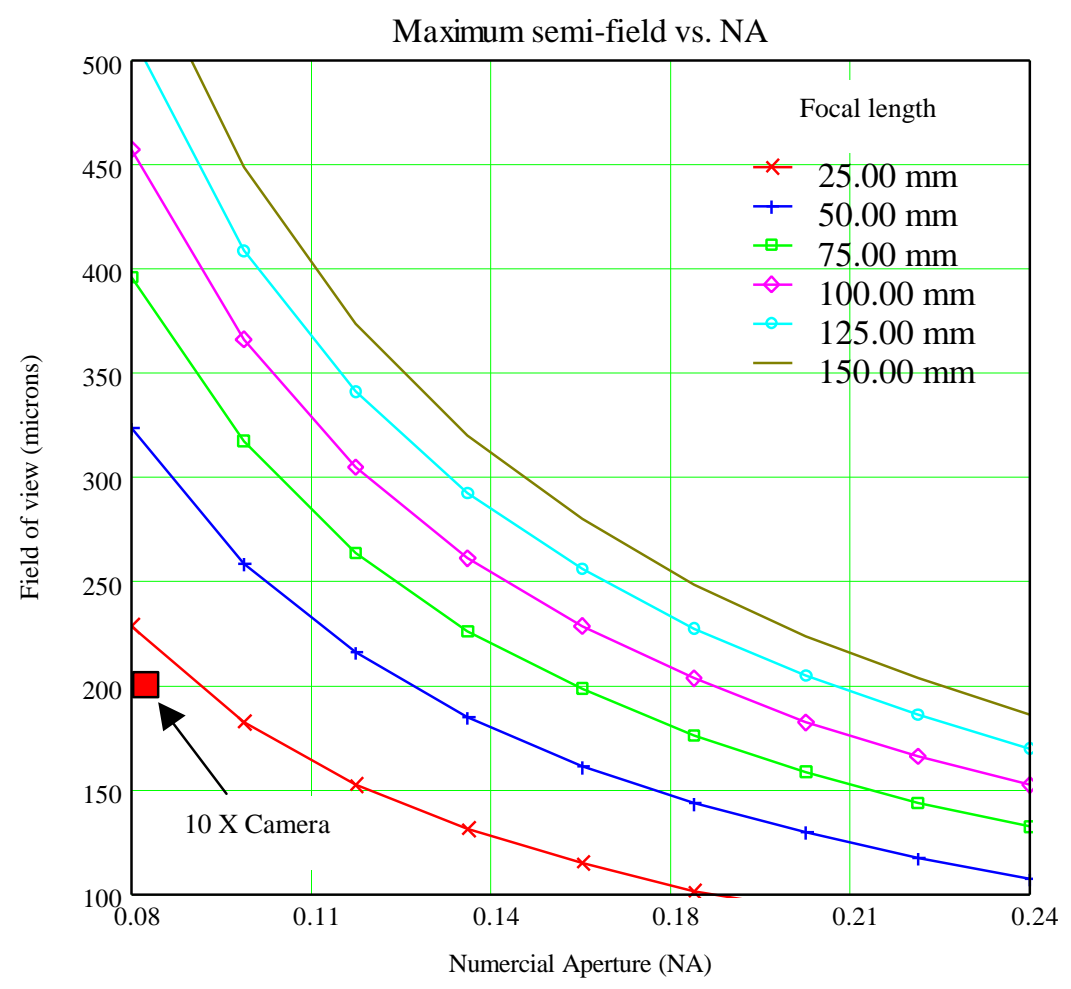

Figure 1 illustrates the maximum semi-field of view of a Schwarzschild objective as a function numerical aperture for a series of increasing focal lengths. Although strictly valid for optical systems whose radii obey the Schwarzschild condition ${ }^{2}$, the curves are a useful estimator of the maximum field of view for any design that is nearly concentric where the ratio of mirror radii (primary/secondary) is in the range of 0.3 to 0.5 . Considering the $10 \mathrm{x}$ camera for example, with a radii ratio of 0.32 and a focal length of 26 $\mathrm{mm}$, the graph predicts that diffraction-limited performance should be achieved across a semi-field of about $230 \mu \mathrm{m}$. In practice, the 10x camera achieves a diffraction-limited field of view of $200 \mu \mathrm{m}$, which is close to the predicted value of $230 \mu \mathrm{m}$.

\subsection{Basic Schwarzschild projection system}

\section{Design for transmission mask}

A camera employing aspheric mirrors in a Schwarzschild configuration is shown in Figure 2. For infinite conjugates, the spherical Schwarzschild is free from spherical aberration, coma, and astigmatism. Locating the stop at the common center of curvature eliminates the distortion as well. In practice, the aperture stop is located at the primary mirror (small mirror). The distortion is not strictly zero but remains well corrected.

${ }^{2}$ Strictly speaking, the Schwarzschild is a concentric, 2-mirror system with a mirror separation $\mathrm{d}=2 \mathrm{f}$, whose radii obey the relations $R_{1}=(\sqrt{ } 5-1) f$ and $R_{2}=(\sqrt{ } 5+1) f$, where $f$ is the focal length of the system. 
The 2-bounce aspheric Schwarzschild in the figure was designed to work at a 5x reduction with a numerical aperture of 0.25 . Even higher numerical apertures are possible. The effective focal length was set to $100 \mathrm{~mm}$. The design was optimized over a linear field of view of $300 \mu \mathrm{m}( \pm 150 \mu \mathrm{m})$ at the wafer. The optical design prescription including the specification data is listed in Appendix A, Table 1. The mirrors are very nearly concentric and nearly obey the Schwarzschild condition $\left[R_{1}=(\sqrt{ } 5-1) f\right.$ and $R_{2}=$ $(\sqrt{ } 5+1) f]$. As shown in the figure, the design can only be used with a transmission mask.

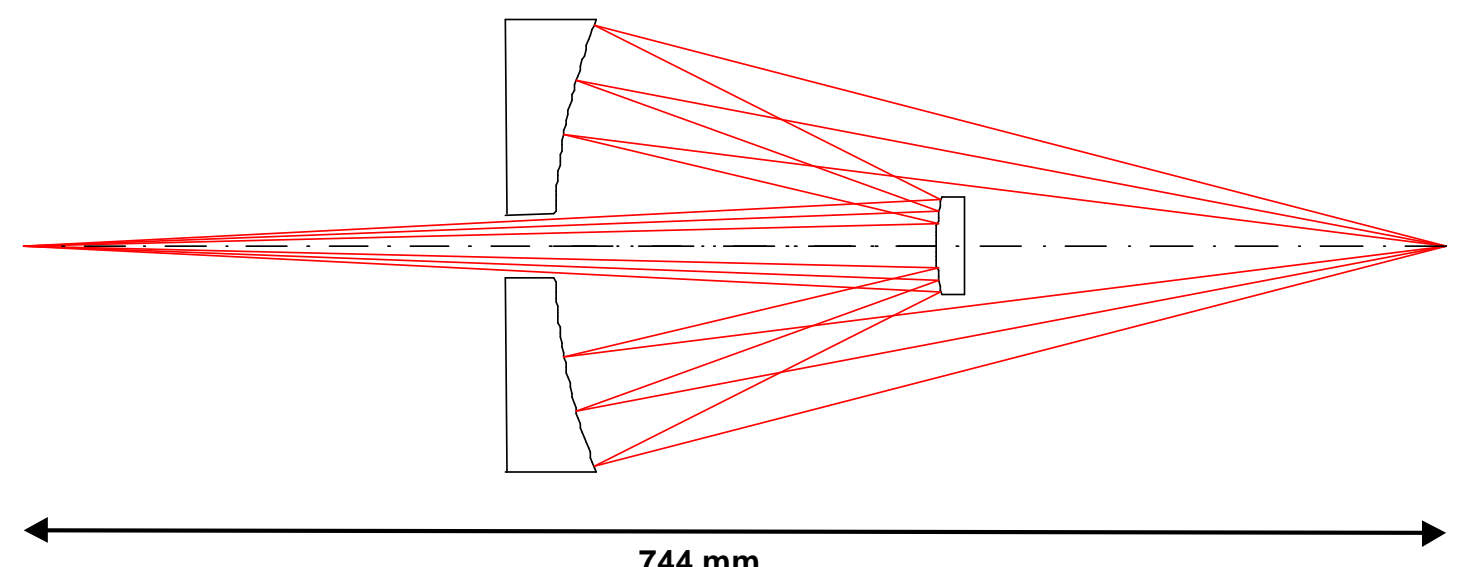

$744 \mathrm{~mm}$

Figure 2. Higher numerical aperture aspheric Schwarzschild for use with a transmission mask. The linear field of view is limited by field curvature to $300 \mu \mathrm{m}( \pm 150 \mu \mathrm{m})$. The design is used at $5 \mathrm{x}$ reduction with a numerical aperture of 0.25 .

This design utilizes elliptical mirrors with $6^{\text {th }}$ and $8^{\text {th }}$ order polynomial deformations to control the aberrations over the expanded NA. The aspheres effectively correct the high-order spherical aberration and coma introduced when the design is used at some finite reduction ratio. The base conics are prolate ellipsoids, so that if the $6^{\text {th }}$ and $8^{\text {th }}$ order deformations are ignored, they are point testable at the their conjugate foci. At a numerical aperture of 0.25 , the design has a composite RMS wavefront error of 0.59 $\mathrm{nm}(0.044 \lambda)$ across the $300 \mu \mathrm{m}$ linear field. Figure 1 shows that by reducing the numerical aperture, the design can support a larger field. At a numerical aperture of 0.10 , the composite RMS wavefront error is $0.64 \mathrm{~nm}(0.048 \lambda)$ across an $800 \mu \mathrm{m}( \pm 400 \mu \mathrm{m})$ linear field at the wafer. In both cases, the design suffers from less than $5 \mathrm{~nm}$ of distortion across the projected format. Since the field of view is small, the chief rays are nearly telecentric at both the mask and wafer. The maximum aspheric departures are $4.3 \mu \mathrm{m}$ and $8.5 \mu \mathrm{m}$ for the primary and secondary mirrors, respectively.

The strong inward-curving field limits this design form. This limitation is severe and a diffraction-limited field of view of only a few hundred microns can be expected for reasonable system lengths $(<750 \mathrm{~mm})$ for a given numerical aperture. Making the mirrors aspheric does nothing (directly) to increase the field of view because field curvature can only be minimized by proper selection of the mirror radii.

The linear obscuration ratio is 0.35 meaning that $12 \%$ of the pupil area is blocked by the primary mirror as the bundle is imaged down to the wafer. This obscuration causes an MTF drop or contrast loss of only 0.12 (MTF drops $0.57 \rightarrow 0.45$ ) for $50 \mathrm{~nm}$ lines and spaces. The design can easily exceed this resolution, but will eventually have difficulty reaching $30 \mathrm{~nm}$ linewidths due to the obscuration.

Increasing the field of view by decreasing the NA leads to a greater obscuration and a resultant loss of contrast that is too great for a "practical" system. The result is nonetheless important since it provides 
insight regarding achievable field size. Fundamentally, the design is limited by field curvature that cannot be overcome with this design form. Figure 3 shows that at a field of $\pm 400 \mu \mathrm{m}$, the actual image surface falls away from the ideal image plane by nearly $2 \mu \mathrm{m}$. The residual field curvature simply uses too much of the available depth of focus.

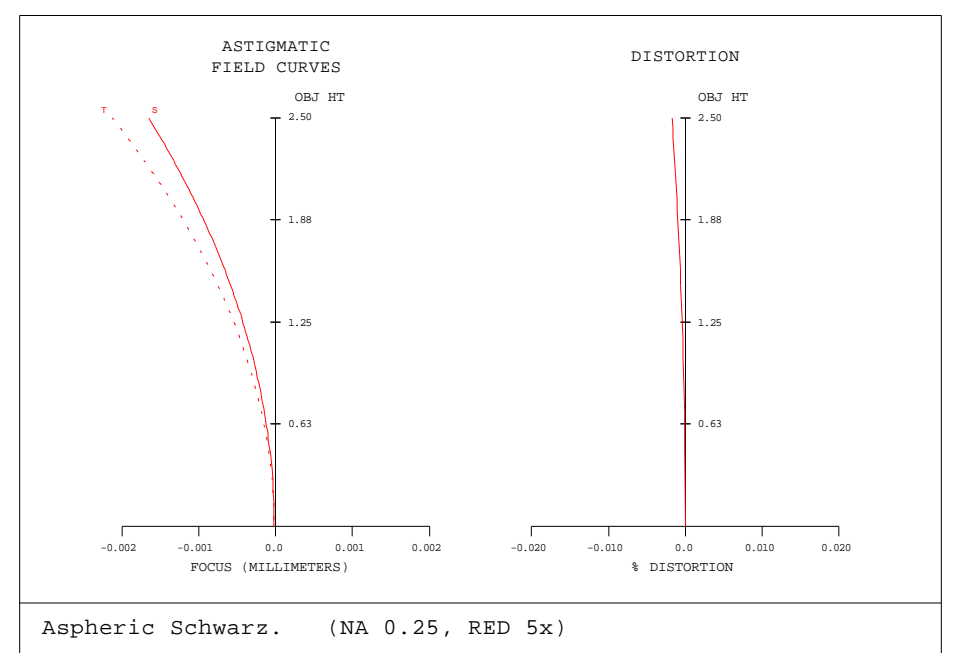

Figure 3. Field curvature of the basic Schwarzschild limits the useable field of view. This plot shows that the image surface actually curves away from the ideal image plane by nearly $2 \mu \mathrm{m}$ at the edge of a $\pm 400 \mu \mathrm{m}$ field at the wafer. Distortion scale has been set so that $0.002 \%$ corresponds to $100 \mathrm{~nm}$.

\section{Design for a reflection mask}

The use of a reflective mask is desirable since we are building on the ETS experience. As with the 10x camera, a reflective mask can be integrated by using an off-axis or eccentric portion of the entrance pupil. Starting with the aspheric centered system, the design was reoptimized for use with a reflective mask (Figure 4). In this case, the pupil is offset by $30 \mathrm{~mm}$ on the primary. To avoid the obscuration, the numerical aperture had to be reduced to 0.125 . A good rule of thumb is that an unobscured design can be made to support one-half of the numerical aperture of its obscured cousin.

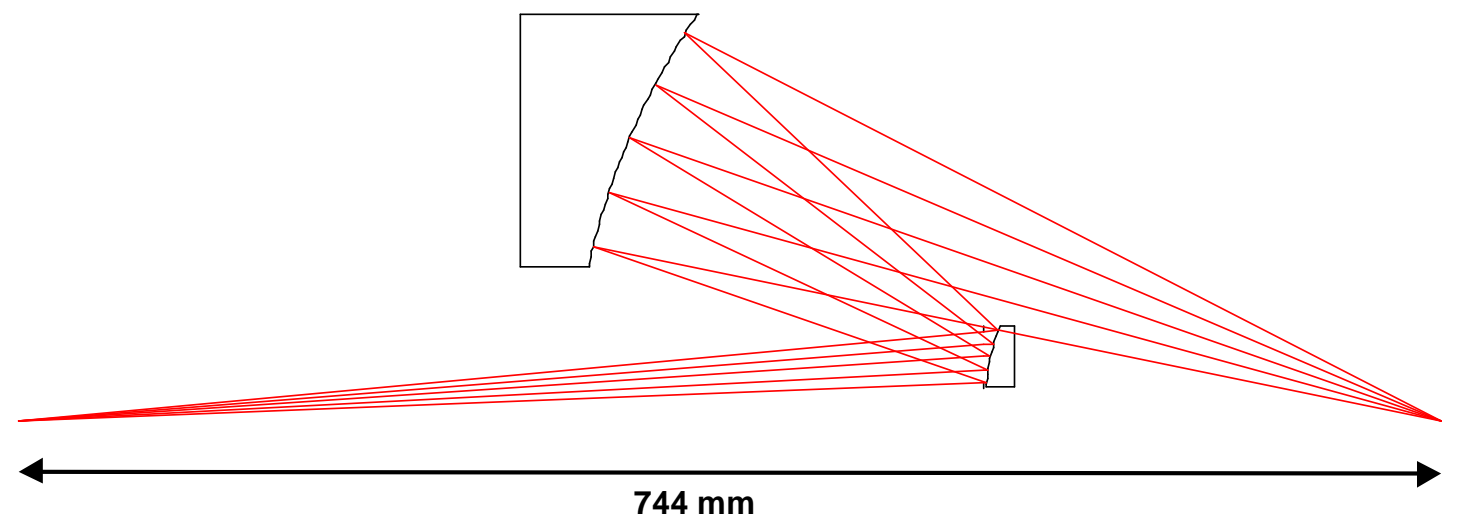

Figure 4. Higher numerical aperture aspheric Schwarzschild for use with a reflective mask. The design has been reoptimized over a rectangular field of $400 \mu \mathrm{m}$ x $200 \mu \mathrm{m}$ at the wafer. The design is used at $5 \mathrm{x}$ reduction with a numerical aperture of 0.125 .

The performance of the off-axis design was rebalanced across a rectangular field that measures $400 \mu \mathrm{m} x$ $200 \mu \mathrm{m}$ at the wafer. The field composite RMS wavefront error is $0.66 \mathrm{~nm}(0.049 \lambda)$. This design 
requires a $\mathrm{k}_{1}$ value of 0.65 to print $70 \mathrm{~nm}$ features $\left(\mathrm{R}=\mathrm{k}_{1} \lambda / \mathrm{NA}\right)$ compared to a $\mathrm{k}_{1}$ value of 0.42 for the current 10x camera. Clearly, this more conservative $\mathrm{k}_{1}$ value will promote better aerial imagery.

The lack of rotational symmetry complicates the behavior of the distortion field so that it cannot be adequately described by a single value. However, the magnitude and orientation of the vector distortion field make this design suitable only for static imaging.

\section{0 “Equal Radii” Projection System}

The field of view of the 10x camera can be scaled up via focal length scaling. The penalty of scaling, however, is that mirror size (and mirror asphericity) increases, resulting in a more difficult mirror fabrication effort. Thus from a practical standpoint, it is good to keep the focal length short so that the mirror clear apertures remain small.

It is worthwhile to investigate alternate design forms. Equation (1) illustrates that $\mathrm{R}_{1}$ needs to be set equal to $\mathrm{R}_{2}$ to achieve the largest possible field. In this manner, the Petzval sum will be corrected and field curvature will not limit the field size. In theory, the field can be made arbitrarily large. This does not happen in practice, since as $\mathrm{R}_{1}$ approaches $\mathrm{R}_{2}$ other factors (aberrations, back focus, ray clearance) start to dominate the design. However, the concept of "equal radii" is a potent design approach for increasing field size.

\section{Design for a transmission mask}

Figure 5 illustrates one of these "equal radii" designs in a coaxial configuration suitable for use with a transmission mask. The design prescription is listed in Appendix A. To achieve a well-corrected image, the design uses ellipsoidal mirrors with $6^{\text {th }}$ order deformations. In this instance $c c>1$ so that both mirrors are oblate spheroids. The aspheric departure is $2.0 \mu \mathrm{m}$ for the primary and $3.0 \mu \mathrm{m}$ for the secondary, respectively. As in the previous case, the design has a numerical aperture of 0.25 and operates at a $5 \mathrm{x}$ reduction. The linear field of view is $1000 \mu \mathrm{m}( \pm 500 \mu \mathrm{m})$ at the wafer. The system is obscured with the final image being formed through a small hole in the primary mirror (a baffle would be needed to prevent light from propagating directly from the mask to the wafer). The distortion is less than $7 \mathrm{~nm}$ across the field and the composite RMS wavefront error is $0.19 \mathrm{~nm}(0.014 \lambda)$. Using this equal radii concept, the field curvature is essentially eliminated. This reduction in field curvature is apparent in Figure 6 and the actual image surface now resides well within the depth of focus.

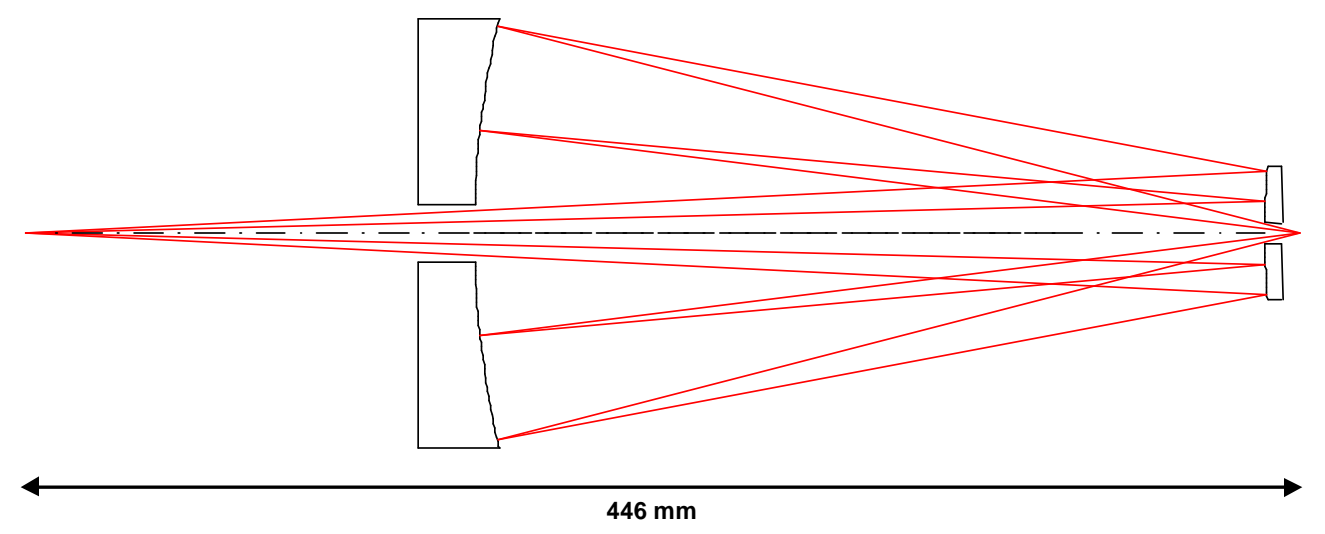

Figure 5. "Equal radii" microstepper concept for use with a transmission mask. The design has a numerical aperture of 0.25 at a reduction of $5 \mathrm{x}$. The field of view is $1 \mathrm{~mm}$ at the wafer. Mirror radii $\mathrm{R} 1$ and $\mathrm{R} 2$ are nearly the same so the Petzval sum is driven to zero. 


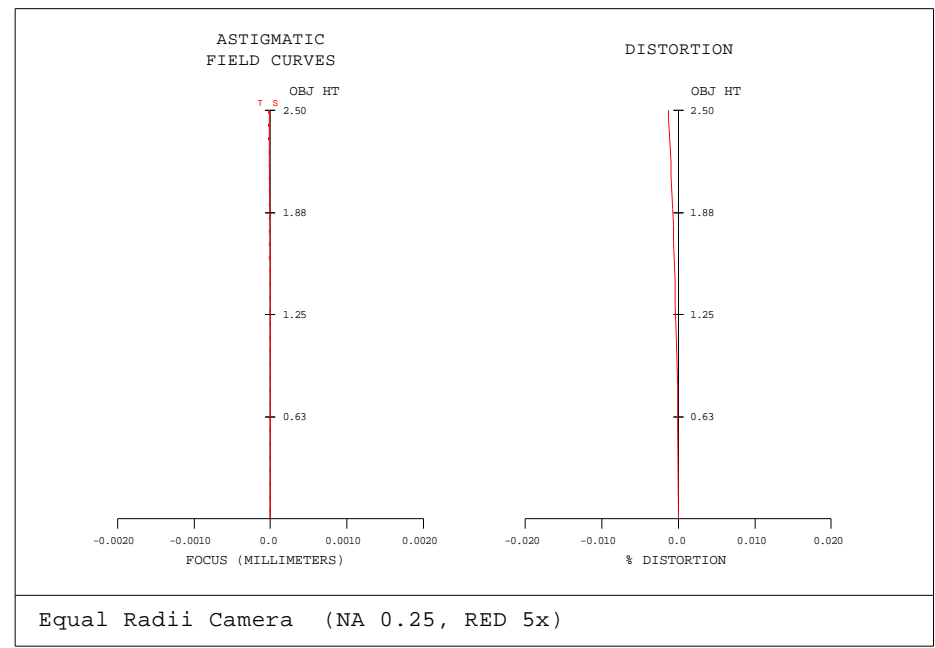

Figure 6. Field curves for the equal radii design. The field curvature is now corrected and the design is limited by astigmatism off axis. Note that the longitudinal separation between the actual image surface and the ideal image plane has been virtually eliminated.

The principle drawback of this approach is the short back working distance. (This illustrates a fundamental trade in microstepper design: allowable field curvature vs. back working distance). For this example, the clearance between the back of the primary and the image plane is $6.35 \mathrm{~mm}$. This distance can be increased by at least a factor of $2 x$, although the increase will probably lead to increased field curvature and obscuration. More investigation is needed to fully quantify the impacts of this trade.

\section{Designs for reflection mask}

Figures 7 and 8 illustrate how the basic centered design can be made to work with a reflective mask. Figure 7 demonstrates the design with a field bias so that the projection system is used in a ring field configuration. The aspheric coefficients were reoptimized to peak performance within this offset field. For simplicity, the projected field at the wafer was made to be an arcuate slit with a radius of $2.25 \mathrm{~mm}$ and a width of $0.40 \mathrm{~mm}$. The field composite RMS wavefront error is $0.70 \mathrm{~nm}(0.052 \lambda)$. The small obscuration has virtually no impact on image contrast for $50 \mathrm{~nm}$ lines and spaces. There are not sufficient degrees of freedom in the design to correct distortion and it grows to about $100 \mathrm{~nm}$ at the edge of the field.

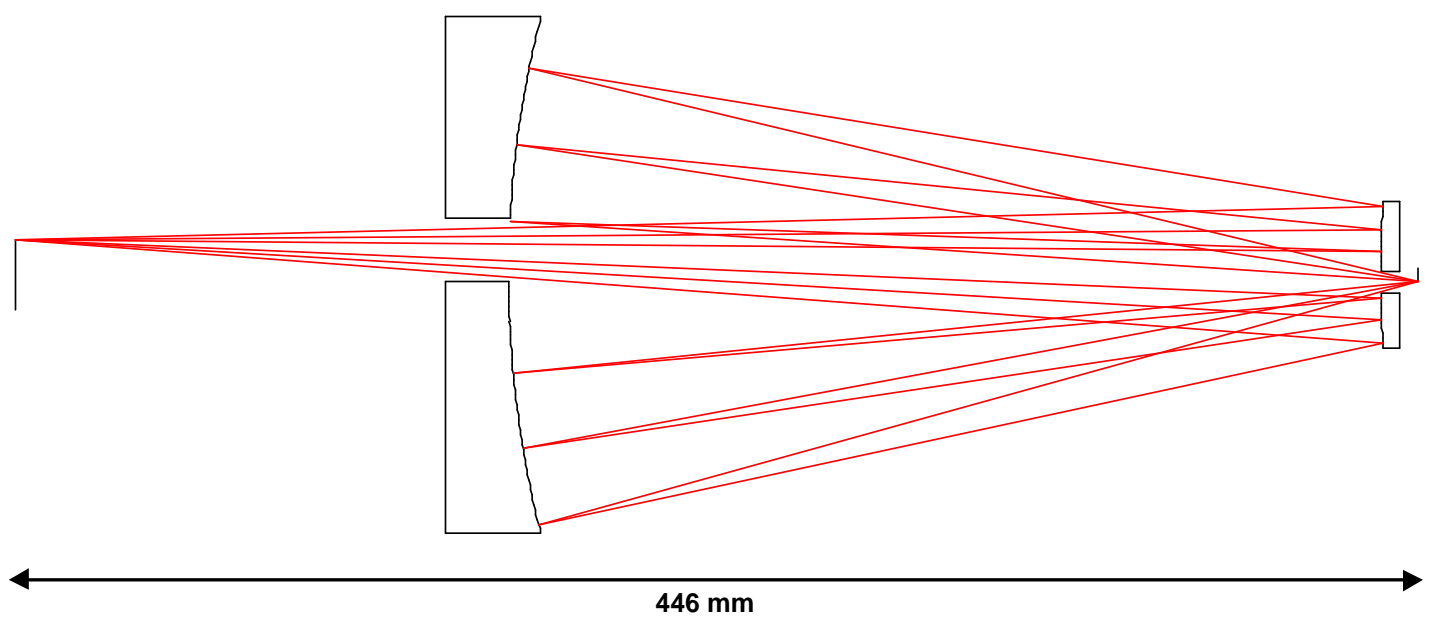

Figure 7. Use of a field bias enables use of a reflection mask. The obscuration is small and it has little impact on the ability to resolve $50 \mathrm{~nm}$ lines and spaces. 


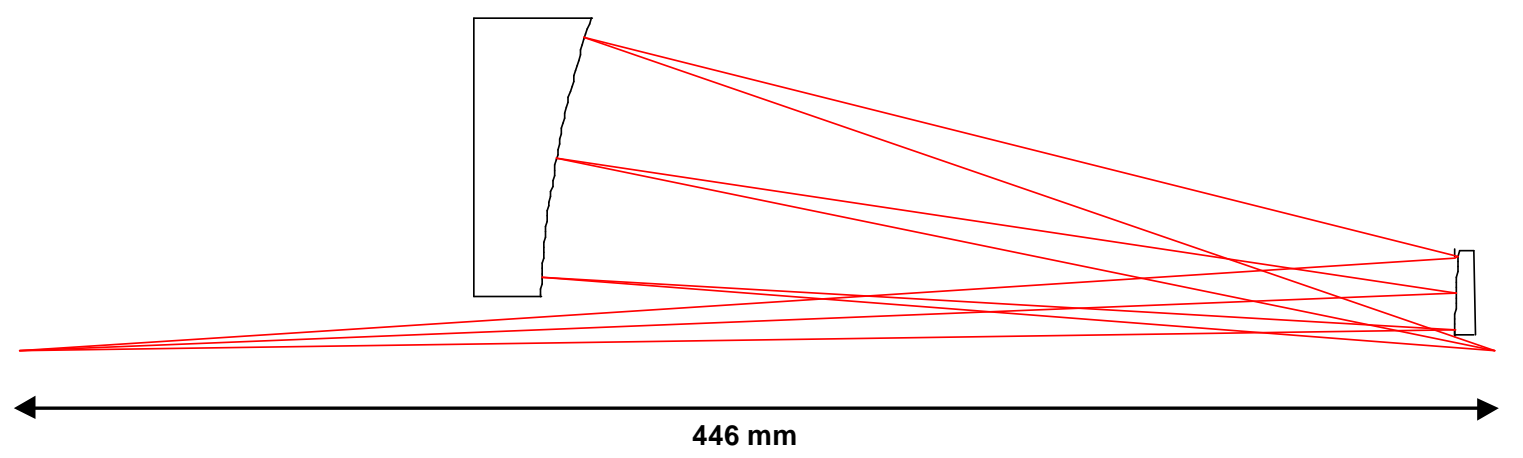

Figure 8. An eccentric pupil allows for unobscured imaging using a reflection mask. The numerical aperture is reduced from 0.25 to 0.125 to add beam clearance near the primary.

Figure 8 demonstrates the time-honored approach of using an offset or eccentric portion of the entrance pupil to accommodate a reflection mask. The eccentric pupil increases the mean chief ray angle to about $9.5^{\circ}$ at the wafer. To allow removal of the obscuration, the numerical aperture is reduced from 0.25 to 0.125 . The aspheric coefficients are reoptimized for peak performance with a $5 \mathrm{x}$ reduction over a rectangular field of $1.0 \mathrm{~mm} \times 0.48 \mathrm{~mm}$ at the wafer. The field composite RMS wavefront error is $0.63 \mathrm{~nm}$ $(0.047 \lambda)$. Compared against a rectilinear grid, the distortion is controlled to better than $9 \mathrm{~nm}$. To test the limits of this design, the field was expanded to $1.5 \mathrm{~mm} \times 0.50 \mathrm{~mm}$ at the wafer and the design was rebalanced. The composite RMS across this field is $0.67 \mathrm{~nm}(0.050 \lambda)$, although the distortion grew to 30 $\mathrm{nm}$ at the corners of the field. For a point of reference, the 10x camera has a composite RMS of $0.74 \mathrm{~nm}$ $(0.055 \lambda)$ as-designed with $100 \mathrm{~nm}$ of distortion at the edge of its $0.28 \mathrm{~mm}$ square field.

\subsection{Conclusion}

The idea of using aspheres in a 2-bounce camera has been proposed in the past, but was not pursued due to risks associated with aspheric mirror fabrication. These risks have been largely mitigated by work associated with the ETS effort, making an aspheric microstepper feasible.

Three practical issues drove this trade study. First, the choice of focal length (system scale) was driven by the PSDI test experience. Note that the mirror radii are close to $300 \mathrm{~mm}$. It is probable that the convex optics can be tested using the current M3 PSDI test set. The concave optics might be tested using modifications to the current M4 test set. Second, the designs are optimized at a reduction of $5 \mathrm{x}$ to accommodate printable defect experiments. Designs at $4 \mathrm{x}$ reduction are possible, but ray clearance starts to become an issue, especially at the mask. Finally, only 2-bounce configurations were examined.

Although both Shafer and Viswanathan ${ }^{3}$ have demonstrated multiple bounces can be used to enhance the level of aberration correction, the as-designed residual wavefront error of our design is small and does not benefit from this technique.

Either the aspheric Schwarzschild or the "equal radii" design is feasible, but the "equal radii" concept is more compelling. The performance of this design is compared to the current 10x microstepper in Table 1 . This design allows an order of magnitude increase in the exposure area at the wafer $\left(0.72 \mathrm{~mm}^{2}\right.$ vs. 0.078 $\mathrm{mm}^{2}$ ) while simultaneously reducing the distortion by a factor of $3 \mathrm{x}(30 \mathrm{~nm}$ vs. $100 \mathrm{~nm})$.

\footnotetext{
${ }^{3}$ See, for example, US Patent No. 5,212,588.
} 
Table 1. Comparison of 10x and "Equal Radii" Camera Parameters

\begin{tabular}{|r|c|c|}
\hline \multicolumn{1}{|c|}{ Parameter } & $10 \mathrm{x}$ & Aspheric “Equal Radii” \\
\hline Mask & Reflection & Reflection \\
\hline Exposure area & $\begin{array}{c}0.078 \mathrm{~mm}^{2} \\
(0.28 \mathrm{~mm} \times 0.28 \mathrm{~mm})\end{array}$ & $\begin{array}{c}0.72 \mathrm{~mm}^{2} \\
(1.5 \mathrm{~mm} \times 0.50 \mathrm{~mm})\end{array}$ \\
\hline NA & 0.080 & 0.125 \\
\hline Focal length & $26 \mathrm{~mm}$ & $100 \mathrm{~mm}$ \\
\hline Reduction & $10 \mathrm{x}$ & $5 \mathrm{x}$ \\
\hline Composite RMS & $0.78 \mathrm{~nm}(0.055 \lambda)$ & $0.67 \mathrm{~nm}(0.050 \lambda)$ \\
\hline Distortion (max) & $100 \mathrm{~nm}$ & $30 \mathrm{~nm}$ \\
\hline $\begin{array}{r}\text { Aspheric } \\
\text { departure }\end{array}$ & None & $2 \mu \mathrm{m} / 3 \mu \mathrm{m}$ \\
\hline Total track & $315.2 \mathrm{~mm}$ & $446.2 \mathrm{~mm}$ \\
\hline $\begin{array}{r}\text { Working } \\
\text { distance }\end{array}$ & $63.7 \mathrm{~mm}$ & $12 \mathrm{~mm}$ \\
\hline
\end{tabular}

It does so with a $10 \%$ improvement in the wavefront error over a $50 \%$ larger numerical aperture. The mirrors have very mild mirror aspheric departure $(2 \mu \mathrm{m}$ departure on the primary and $3 \mu \mathrm{m}$ departure on the secondary). The disadvantage of this design is the short back working distance $(63.7 \mathrm{~mm}$ vs. $12 \mathrm{~mm}-$ defined from the vertex of the primary to the mask plane). However, it appears feasible that this distance can be increased to match that of contemporary DUV steppers. 


\section{Appendix A. Optical design prescriptions}

Table 1. Centered aspheric Schwarzschild prescription

\begin{tabular}{|c|c|c|c|c|}
\hline \multicolumn{3}{|r|}{ RDY } & THI & \multirow[t]{2}{*}{ GLA } \\
\hline OBJ : & \multicolumn{2}{|c|}{ INFINITY } & 277.587081 & \\
\hline $1:$ & \multicolumn{2}{|c|}{ INEINITY } & 200.000000 & \multirow[b]{2}{*}{ REFL } \\
\hline STO: & \multicolumn{2}{|c|}{115.54978} & -200.000000 & \\
\hline \multicolumn{5}{|c|}{ ASP : } \\
\hline $\mathrm{K}$ & -0 & \multicolumn{3}{|c|}{-0.555172} \\
\hline B & 0 & $0.141231 \mathrm{E}-11$ & \multicolumn{2}{|c|}{$\mathrm{C} \quad:-.526934 \mathrm{E}-15$} \\
\hline 3: & & 326.76288 & 200.000000 & REFL \\
\hline \multicolumn{5}{|c|}{ ASP : } \\
\hline $\mathrm{K}$ & -0 & \multicolumn{3}{|c|}{-0.050088} \\
\hline B & 0 & $0.148261 \mathrm{E}-15$ & \multicolumn{2}{|c|}{$\mathrm{C}:-.434588 \mathrm{E}-21$} \\
\hline $4:$ & & INFINITY & \multicolumn{2}{|c|}{266.171149} \\
\hline IMG : & & NFINITY & \multicolumn{2}{|l|}{0.000000} \\
\hline \multicolumn{5}{|c|}{ SPECIFICATION DATA } \\
\hline NAO & & 0.05000 & & \\
\hline DIM & & $\mathrm{MM}$ & & \\
\hline WL & & 13.40 & & \\
\hline $\mathrm{REF}$ & & 1 & & \\
\hline WTW & & 1 & & \\
\hline $\mathrm{XOB}$ & & 0.00000 & 0.00000 & \\
\hline YOB & & 0.00000 & 0.75000 & \\
\hline
\end{tabular}

Table 2. Centered "Equal Radii" design prescription

\begin{tabular}{|c|c|c|c|c|c|}
\hline & \multicolumn{2}{|r|}{ RDY } & \multicolumn{2}{|c|}{ THI } & GLA \\
\hline OBJ : & \multicolumn{2}{|c|}{ INF INITY } & \multicolumn{2}{|c|}{434.199856} & \\
\hline STO: & \multicolumn{2}{|c|}{327.80576} & \multirow{2}{*}{\multicolumn{2}{|c|}{-277.006762}} & REFL \\
\hline \multicolumn{4}{|c|}{ ASP : } & & \\
\hline $\mathrm{K}$ & \multicolumn{2}{|c|}{10.395994} & \multirow{2}{*}{\multicolumn{3}{|c|}{$:-.651934 \mathrm{E}-12$}} \\
\hline A & $: 0.000$ & $000 \mathrm{E}+00$ & & & \\
\hline \multirow{2}{*}{ 2: } & \multicolumn{2}{|c|}{334.14538} & \multirow{2}{*}{\multicolumn{2}{|c|}{277.006762}} & REFL \\
\hline & \multicolumn{3}{|c|}{ ASP : } & & \\
\hline $\mathrm{K}$ & \multicolumn{2}{|c|}{0.091842} & \multirow{2}{*}{\multicolumn{3}{|c|}{$: 0.518232 E-15$}} \\
\hline A & \multicolumn{2}{|c|}{$: 0.000000 \mathrm{E}+00$} & & & \\
\hline $3:$ & \multicolumn{2}{|c|}{ INFINITY } & \multicolumn{3}{|c|}{12.000000} \\
\hline IMG : & & INE INITY & \multicolumn{3}{|c|}{0.000000} \\
\hline \multicolumn{3}{|c|}{ SPECIFICATION DATA } & & & \\
\hline $\mathrm{NAO}$ & & 0.05000 & & & \\
\hline DIM & & $\mathrm{MM}$ & & & \\
\hline WL & & 13.40 & & & \\
\hline $\mathrm{REF}$ & & 1 & & & \\
\hline WTW & & 1 & & & \\
\hline $\mathrm{XOB}$ & & 0.00000 & 0.00000 & 0 & 000 \\
\hline YOB & & 0.00000 & 1.00000 & 2.0 & 00 \\
\hline
\end{tabular}

